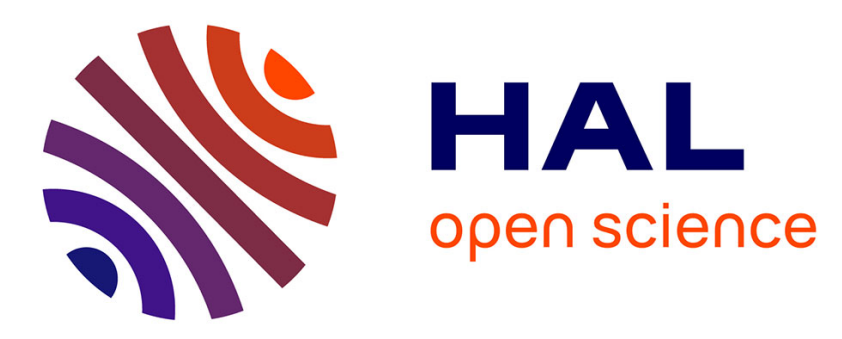

\title{
Automated Symbolic Proofs of Observational Equivalence
}

David Basin, Jannik Dreier, Ralf Sasse

\section{To cite this version:}

David Basin, Jannik Dreier, Ralf Sasse. Automated Symbolic Proofs of Observational Equivalence. 22nd ACM SIGSAC Conference on Computer and Communications Security (ACM CCS 2015), ACM, Oct 2015, Denver, United States. pp.1144-1155, 10.1145/2810103.2813662 . hal-01337409v2

\section{HAL Id: hal-01337409 https://hal.science/hal-01337409v2}

Submitted on 12 Sep 2017

HAL is a multi-disciplinary open access archive for the deposit and dissemination of scientific research documents, whether they are published or not. The documents may come from teaching and research institutions in France or abroad, or from public or private research centers.

$$
\text { Copyright }
$$

L'archive ouverte pluridisciplinaire HAL, est destinée au dépôt et à la diffusion de documents scientifiques de niveau recherche, publiés ou non, émanant des établissements d'enseignement et de recherche français ou étrangers, des laboratoires publics ou privés. 


\section{Automated Symbolic Proofs of Observational Equivalence}

\author{
David Basin \\ Inst. of Information Security \\ Dept. of Computer Science \\ ETH Zurich, Switzerland \\ basin@inf.ethz.ch
}

\author{
Jannik Dreier \\ Inst. of Information Security \\ Dept. of Computer Science \\ ETH Zurich, Switzerland \\ jannik.dreier@inf.ethz.ch
}

\author{
Ralf Sasse \\ Inst. of Information Security \\ Dept. of Computer Science \\ ETH Zurich, Switzerland \\ ralf.sasse@inf.ethz.ch
}

\begin{abstract}
Many cryptographic security definitions can be naturally formulated as observational equivalence properties. However, existing automated tools for verifying the observational equivalence of cryptographic protocols are limited: they do not handle protocols with mutable state and an unbounded number of sessions. We propose a novel definition of observational equivalence for multiset rewriting systems. We then extend the TAMARIN prover, based on multiset rewriting, to prove the observational equivalence of protocols with mutable state, an unbounded number of sessions, and equational theories such as Diffie-Hellman exponentiation. We demonstrate its effectiveness on case studies, including a stateful TPM protocol.
\end{abstract}

\section{Categories and Subject Descriptors}

D.2.4 [Software/Program Verification]: Formal methods; K.4.4 [Electronic Commerce]: Security

\section{General Terms}

Security, Verification

\section{Keywords}

Protocol verification, observational equivalence, symbolic model

\section{INTRODUCTION}

Security protocols are the backbone of secure communication in open networks. It is well known that their design is error-prone and formal proofs can increase confidence in their correctness. Most tool-supported proofs have focused on trace properties, like secrecy as reachability and authentication as correspondence. But observational equivalence has received increasing attention and it is frequently used to express security properties of cryptographic protocols. Examples include stronger notions of secrecy and privacy-related properties of voting and auctions $[14,16,17,18]$, gamebased notions such as ciphertext indistinguishability [5], and authenticated key-exchange security $[6,21]$.

Our focus in this paper is on symbolic models [4] for observational equivalence. The key advantage of using a symbolic model is that it enables a higher degree of automation in tools $[9,11,8,7,29]$ for protocol analysis. These tools can quickly find errors in protocols or demonstrate their correctness with respect to symbolic abstractions. Moreover, they do not require a manual, tedious, and error-prone proof for each protocol. Unfortunately, none of the above tools are capable of analyzing protocols with mutable state for an unbounded number of sessions with respect to a security property based on observational equivalence. Note that mutable state is a key ingredient for many kinds of protocols and systems, for example to specify and analyze security APIs for hardware security modules [22].

In this paper, we develop a novel and general definition of observational equivalence in the symbolic setting of multiset rewriting systems. We present an algorithm suitable for protocols with mutable state, an unbounded number of sessions, as well as equational properties of the cryptographic operations, such as Diffie-Hellman exponentiation. Our algorithm is sound but not complete, yet it succeeds on a large class of protocols. We illustrate this through case studies using our implementation of the algorithm in the TAMARIN prover.

As case studies we verify the untraceability of an RFID protocol, and find an attack on the TPM_Envelope protocol when using deterministic encryption. Note that some protocols, such as TPM_Envelope, have been analyzed before with symbolic methods [15]. However, their analyses were carried out with respect to weaker trace-based security properties such as the unreachability of a state where the adversary can derive secrets. Formulating security properties in terms of observational equivalence is much closer to the properties used in game-based cryptographic proofs than trace properties are. For example, game-based protocol analysis often uses the standard test [24] of distinguishing real-or-random, where the adversary is unable to distinguish the real secret from an unrelated randomly generated value.

Contribution. We give a novel definition of observational equivalence in the multiset rewriting framework and an associated algorithm which is the first that supports mutable state, an unbounded number of sessions, and Diffie-Hellman exponentiation. We implement this algorithm in an extension of the TAMARIN prover and we demonstrate its practicality in different case studies that illustrate its features. The resulting proofs are largely automated, with limited 
manual input needed to select proof strategies in some cases.

Structure. We introduce our general system model based on multiset rewriting in Section 2. We motivate and define observational equivalence for multiset rewrite systems in Section 3. We show how to prove observational equivalence in Section 4 and sketch its implementation in the TAMARIN prover. Afterwards we present our case studies in Section 5. We close with related work and draw conclusions in Section 6.

\section{PRELIMINARIES AND MODEL}

Let $S^{*}$ denote the set of sequences over $S$. For a sequence $s$, we write $s_{i}$ for its $i$-th element, $|s|$ for its length, and $i d x(s)=\{1, \ldots,|s|\}$ for the set of its indices. We use [] to denote the empty sequence, $\left[s_{1}, \ldots, s_{k}\right]$ to denote the sequence $s$ of length $k$, and $s \cdot s^{\prime}$ to denote the concatenation of the sequences $s$ and $s^{\prime}$.

We specify properties of functions by equations. Given a signature $\Sigma_{F u n}$ of functions and a set $V$ of variables, $T_{\Sigma_{F u n}}(V)$ denotes the set of terms built using functions from $\Sigma_{F u n}$ and variables from $V$. Terms without variables are called ground terms and denoted $T_{\Sigma_{F u n}}$. An equation over the signature $\Sigma_{F u n}$ is an unordered pair of terms $s, t \in T_{\Sigma_{F u n}}(V)$, written $s \simeq t$. An equational presentation is a pair $\mathcal{E}=\left(\Sigma_{F u n} ; E\right)$ of a signature $\Sigma_{F u n}$ and a set of equations $E$. The corresponding equational theory $=\mathcal{E}$ is the smallest $\Sigma_{F u n}$-congruence containing all instances of the equations in $E$. We often leave the signature $\Sigma_{F u n}$ implicit and identify the equations $E$ with the equational presentation $\mathcal{E}$. Similarly, we use $={ }_{E}$ for the equational theory $=_{\mathcal{E}}$. We say that two terms $s$ and $t$ are equal modulo $E$ iff $s={ }_{E} t$. We use the subscript $E$ to denote the usual operations on sets, sequences, and multisets where equality is modulo $E$ instead of syntactic equality. For example, we write $\epsilon_{E}$ for set membership modulo $E$.

EXAMPLE 1. To model symmetric key encryption, let $\Sigma_{\text {Fun }}$ be the signature consisting of the functions enc $(\cdot, \cdot)$ and $\operatorname{dec}(\cdot, \cdot)$ together with the equation $\operatorname{dec}(\operatorname{enc}(x, k), k) \simeq x$.

We model systems using multiset rewrite rules. These rules manipulate multisets of facts which model the current state of the system, with terms as arguments. Formally, given a signature $\Sigma_{F u n}$ and a (disjoint) set of fact symbols $\Sigma_{\text {Fact }}$, we define $\Sigma=\Sigma_{\text {Fun }} \cup \Sigma_{\text {Fact }}$, and we define the set of facts as $\mathcal{F}=\left\{F\left(t_{1}, \ldots, t_{n}\right) \mid t_{i} \in T_{\Sigma_{\text {Fun }}}, F \in \Sigma_{\text {Fact }}\right.$ of arity $\left.n\right\}$. We assume that $\Sigma_{\text {Fact }}$ is partitioned into linear and persistent fact symbols; a fact $F\left(t_{1}, \ldots, t_{n}\right)$ is called linear if its function symbol $F$ is linear, and persistent if $F$ is persistent. Linear facts model resources that can only be consumed once, whereas persistent facts can be consumed as often as needed. We denote by $\mathcal{F}^{\sharp}$ the set of finite multisets built using facts from $\mathcal{F}$, and by $\mathcal{G}^{\sharp}$ the set of multisets of ground facts.

The system's possible state transitions are modeled by labeled multiset rewrite rules. A labeled multiset rewrite rule is a tuple $(i d, l, a, r)$, written $i d: l-\left[a \longmapsto r\right.$, where $l, a, r \in \mathcal{F}^{\sharp}$ and $i d \in \mathcal{I}$ is a unique identifier. Given a rule $r i=i d$ : $l-\{a\} r$, name $($ ri $)=i d$ denotes its name, prems $(r i)=l$ its premises, acts $(r i)=a$ its actions, and $\operatorname{concs}(r i)=r$ its conclusions. Finally ginsts $(R)$ denotes the ground instances of a set $R$ of multiset rewrite rules, ffacts $(l)$ is the multiset of all linear facts in $l$, and pfacts $(l)$ is the set of all persistent facts in $l$. We use $m \operatorname{set}(s)$ to highlight that $s$ is a multiset, and we use $\operatorname{set}(s)$ for the interpretation of $s$ as a set, even if it is a multiset. We use regular set notation $\{\cdot\}$ for multisets as well, whenever it is clear from the context whether it is a set or a multiset.

EXAMPLE 2. The following multiset rewrite rules describe a system that constructs terms containing nested applications of the functions one $(\cdot)$ and two $(\cdot)$ inside a fact built with the symbol $M$ using the first three rules below. Using the final rule, $E_{\text {check }}$, the system can compare a constructed term with the value stored in the $\operatorname{In}_{E n v}(\cdot)$ fact.

$$
\begin{aligned}
& \text { Env }=\left\{E_{\text {null }}:-[\} \rightarrow M(\text { null }),\right. \\
& E_{\text {one }}: M(x)-[] \rightarrow M(\text { one }(x)) \text {, } \\
& E_{t w o}: M(x)-[\rightarrow M(t w o(x)) \text {, } \\
& \left.E_{\text {check }}: M(x), \operatorname{In}_{\text {Env }}(x)-[\} \text { Out }_{\text {Env }}(\text { true })\right\}
\end{aligned}
$$

In our semantics of multiset rewriting, we associate each fact $F$ with a recipe recipe $(F)$, representing how this fact was derived. This will be important for defining observational equivalence later. Specifically, we define a sequence of the premises $s e q^{\leq}(l)$ and conclusions $s e q^{\leq}(r)$ of a rule $i d: l-\{a \mapsto r$ by ordering all facts under the total order $\leq$. Usually, $\leq$ will just be the lexicographic order, where if the same fact symbol appears repeatedly, we order the instances of each such fact lexicographically by the terms inside the fact. If these terms are also identical, the facts can appear in any order. Given a rule $i d: l-\{a \nmid r$, for a fact $F \in r$, where $k$ is the index of $F$ in seq $\leq(r)$, and $l_{1}, \ldots, l_{n}=s e q \leq(l)$, we have

$$
\operatorname{recipe}(F)=i d_{k}\left(\operatorname{newvars}(F),\left[\operatorname{recipe}\left(l_{1}\right), \ldots, \operatorname{recipe}\left(l_{n}\right)\right]\right),
$$

where newvars $(F)$ denotes the list of new variables. New variables are those that appear in $F$ but not in any of the premises. Thus, we include their instantiations, e.g., $[\{a / x\}]$ for the list containing the new variable $x$ instantiated with $a$. This list is ordered by the appearance of the new variables inside $F$. This definition requires computing the recipes for the facts $l_{1}, \ldots, l_{n}$ recursively. Moreover, by abuse of notation, we define the recipe of a rule $i d$ as

$$
\begin{aligned}
\operatorname{recipe}(i d)= & i d\left(\left[\operatorname{newvars}\left(r_{1}\right), \ldots, \operatorname{newvars}\left(r_{m}\right)\right],\right. \\
& {\left.\left[\operatorname{recipe}\left(l_{1}\right), \ldots, \operatorname{recipe}\left(l_{n}\right)\right]\right), }
\end{aligned}
$$

where $r_{1}, \ldots, r_{m}=s e q \leq(r)$. It consists of the list of lists of new variables and the list of all recipes of the premises. We denote by $\rho$ the set of all recipes of rules.

The semantics of a set of multiset rewrite rules $P$ are given by a labeled transition relation $\rightarrow_{P} \subseteq \mathcal{G}^{\sharp} \times\left(\mathcal{G}^{\sharp} \times \rho\right) \times \mathcal{G}^{\sharp}$, defined by the transition rule:

$$
\begin{aligned}
& r i=i d: l-\{a\rceil r \in_{E} \text { ginsts }(P)
\end{aligned}
$$

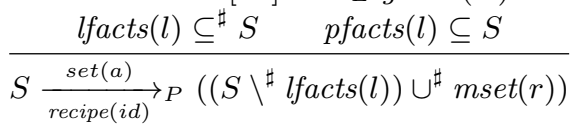

Note that the initial state of an LTS derived from multiset rewrite rules is the empty set of facts $\emptyset$. Each transition transforms a multiset of facts $S$ into a new multiset of facts, according to the rewrite rule used. Moreover each transition is labeled by the actions $a$ of the rule, as well as the rule's recipe recipe $(i d)$. These labels are used in our definition of observational equivalence below, for example that each interface transition must be simulated by the same transition. Since we perform multiset rewriting modulo $E$, we use $\epsilon_{E}$ 


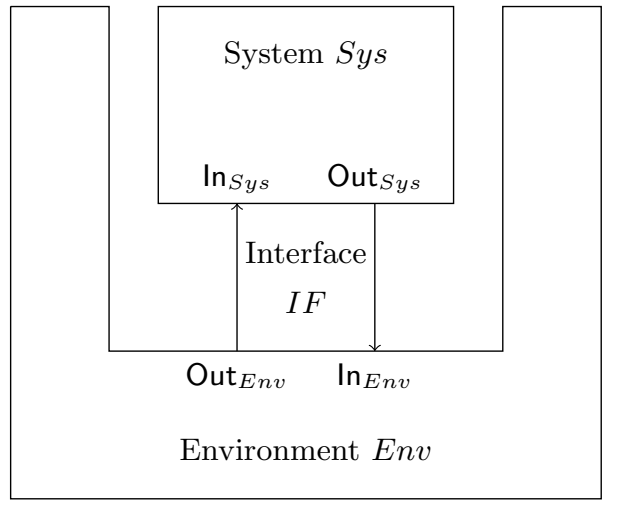

Figure 1: System model

for the rule instance. As linear facts are consumed upon rewriting, we use multiset inclusion, written $\subseteq$, to check that all facts in lfacts $(l)$ occur sufficiently often in $S$. For persistent facts, we only check that each fact in pfacts $(l)$ occurs in $S$. To obtain the successor state, we remove the consumed linear facts and add the generated facts.

EXAmple 3 (PAIRs). Consider two systems, where the first system outputs a pair of identical values

$$
S_{A}=\left\{A:-[\} \text { Out }_{\text {Sys }}((x, x))\right\}
$$

and the second system may output two different values

$$
S_{B}=\left\{B:-[\} \text { Out }_{\text {Sys }}((x, y))\right\} .
$$

In $S_{A}$, we have that

$$
\emptyset \underset{A([\{m / x\}],[])}{\longrightarrow}\left\{\text { Out }_{\text {Sys }}((m, m))\right\} .
$$

In $S_{B}$, we can either take a similar transition

$$
\emptyset \underset{B([\{m / x, m / y\}],[])}{\longrightarrow}\left\{\text { Out }_{\text {Sys }}((m, m))\right\}
$$

or alternatively

$$
\emptyset \underset{B([\{m / x, n / y\}],[])}{\longrightarrow}\left\{\text { Out }_{\text {Sys }}((m, n))\right\} .
$$

\section{OBSERVATIONAL EQUIVALENCE}

Observational equivalence expresses that two systems appear the same to the environment. This can be used to specify security properties such as the inability of an attacker to distinguish between two instances of a protocol. It also has applications in system verification, for example in formalizing that the environment sees no difference between interacting with an ideal system or a concrete implementation. To define observational equivalence, we must model the system, the environment, and their interface.

In our model, depicted in Fig. 1, we model both the System Sys and the environment Env using multiset rewrite rules. We require that the sets of facts and rules used by the system and the environment are disjoint, and that their signatures provide "communication facts" Out ${ }_{S y s}, \ln _{S y s}$, Out $_{E n v}$, and $\ln _{E n v}$ as an interface for interaction. Their interaction is described by the following interface rules.

$$
\begin{aligned}
O U T & =\left\{O U T: \operatorname{Out}_{S y s}(M)-[\mathrm{O}\} \ln _{E n v}(M)\right\} \\
I N & =\left\{I N: \operatorname{Out}_{E n v}(M)-[\mathrm{I}\} \operatorname{In}_{S y s}(M)\right\} \\
I F & =O U T \cup I N
\end{aligned}
$$

The OUT rule forwards the system's output to the environment's input and the IN rule forwards the environment's output to the system's input.

In our interface rules, each input and output is labeled using the action $\mathrm{O}$, for system output, or $\mathrm{I}$, for system input. We model that the environment can only observe these interactions, but not the internal state or transitions within the system, which should be invisible to the environment. We reflect this in the recipes by defining the recipe of the $\ln _{E n v}(M)$ fact as a conclusion of the OUT-rule differently from other facts in the system or environment rules. Namely, we define recipe $\left(\operatorname{In}_{E n v}(M)\right)=O U T_{1}([], x)$, where $x$ is a new variable. Similarly we define the recipe of the rule as recipe $(O U T)=O U T([], x)$. This replaces the recipe of the Out $_{\text {Sys }}(M)$ fact, which is considered to be internal to the system, with a variable. Note that this replacement makes the book-keeping of recipes inside the system unnecessary; however we keep them in our formalization as it simplifies the definition of the LTS as we therefore do not need to distinguish between system and environment transitions.

EXAmple 4 (PAirs Revisited). Consider the two systems from Example 3 and the following environment, which can check whether the two values in a pair are equal:

$$
E n v=\left\{C: \ln _{E n v}(x, x)-[\} \text { Out }_{E n v}(\text { true })\right\} .
$$

Then in $S_{A} \cup I F \cup$ Env we have

$$
\begin{aligned}
\emptyset & \underset{A([\{m / x\}],[])}{\longrightarrow}\left\{\text { Out }_{\text {Sys }}((m, m))\right\} \\
& \underset{\text { OUT }([], z)}{\longrightarrow}\left\{\operatorname{In}_{E n v}((m, m))\right\} \\
& \underset{C\left([],\left[\text { OUT }_{1}([], z)\right]\right)}{\longrightarrow}\left\{\text { Out }_{\text {Env }}(\text { true })\right\} .
\end{aligned}
$$

In $S_{B} \cup I F \cup$ Env we have similar transitions:

$$
\begin{aligned}
\emptyset & \underset{B([\{m / x, m / y\}],[])}{\longrightarrow}\left\{\text { Out }_{\text {Sys }}((m, m))\right\} \\
& \underset{O}{\stackrel{O U T([], z)}{\longrightarrow}}\left\{\operatorname{In}_{E n v}((m, m))\right\} \\
& \underset{C\left([],\left[\text { OUT }_{1}([], z)\right]\right)}{\longrightarrow}\left\{\text { Out }_{E n v}(\text { true })\right\} .
\end{aligned}
$$

Note that the first transition has a different recipe as we also must instantiate $y$, but the output replaces this recipe with a new variable $z$, which is the same on both sides. This hides from the environment how the term was constructed. However, in $S_{B} \cup I F \cup$ Env we also have the following transitions:

$$
\begin{aligned}
\emptyset & \underset{B([\{m / x, n / y\}],[])}{\longrightarrow}\left\{\text { Out }_{\text {Sys }}((m, n))\right\} \\
& \underset{O}{\operatorname{OUT}([], z)}\left\{\operatorname{In}_{E n v}((m, n))\right\} .
\end{aligned}
$$

Note that the first transition has a different recipe as we instantiate $y$ differently, but again the output replaces this recipe with a new variable $z$, which is the same on both sides.

\subsection{Definition}

We formalize observational equivalence in the context of labeled transition systems (LTS) induced by two multiset rewrite systems. Since the environment does not know with which system it is interacting, each transition caused by an environment rule must be matched by the same rule operating on facts with the same recipes, ensuring that the environment makes the same choices in both cases. Otherwise the environment could trivially distinguish any two systems by choosing transitions depending on which system it interacts with. Similarly, all interface rules must be matched by 
themselves, ensuring that an input can only be matched by an input, and an output only by an output.

In contrast to the above, transitions inside one system can be matched by any number of transitions in the other system since the environment cannot observe these steps. This is reflected by the OUT rule, where the recipe of a system output is removed, ensuring that the environment does not know how the term was constructed. Note that this still allows the environment to distinguish both systems if their behavior on a given input differs, or if they output terms that can be distinguished by the environment. An example of the latter is when a transition in the environment requiring the equality of two terms with the same recipes (i.e., deduced using the same steps from the same outputs) is possible in one system, but not in the other.

Definition 1 (ObSERVATIONAL Equivalence $\approx$ ).

Two sets of multiset rewrite rules $S_{A}$ and $S_{B}$ are observational equivalent with respect to an environment given by a set of multiset rewrite rules Env, written $S_{A} \approx_{E n v} S_{B}$, if, given the LTS defined by the rules $S_{A} \cup I F \cup$ Env and $S_{B} \cup I F \cup$ Env, there is a relation $\mathcal{R}$ containing the initial states, such that for all states $\left(\mathcal{S}_{A}, \mathcal{S}_{B}\right) \in \mathcal{R}$ we have:

1. If $\mathcal{S}_{A} \underset{r}{\stackrel{l}{\rightarrow}} \mathcal{S}_{A}^{\prime}$ and $r$ is the recipe of a rule in Env $\cup I F$, then there exists actions $l^{\prime} \in \mathcal{F}^{\sharp}$ and $\mathcal{S}_{B}^{\prime} \in \mathcal{G}^{\sharp}$ such that $\mathcal{S}_{B} \underset{r}{\stackrel{l^{\prime}}{\longrightarrow}} \mathcal{S}_{B}^{\prime}$, and $\left(\mathcal{S}_{A}^{\prime}, \mathcal{S}_{B}^{\prime}\right) \in \mathcal{R}$.

2. If $\mathcal{S}_{A} \underset{r}{\stackrel{l}{\rightarrow}} \mathcal{S}_{A}^{\prime}$ and $r$ is the recipe of a rule in $S_{A}$, then there exist recipes $r_{1}, \ldots, r_{n} \in \rho$ of rules in $S_{B}$, actions $l_{1}, \ldots, l_{n} \in \mathcal{F}^{\sharp}, n \geq 0$, and $\mathcal{S}_{B}^{\prime} \in \mathcal{G}^{\sharp}$ such that $\mathcal{S}_{B} \underset{r_{1}}{\stackrel{l_{1}}{\longrightarrow}}$ $\ldots \underset{r_{n}}{\stackrel{l_{n}}{\longrightarrow}} \mathcal{S}_{B}^{\prime}$, and $\left(\mathcal{S}_{A}^{\prime}, \mathcal{S}_{B}^{\prime}\right) \in \mathcal{R}$.

Additionally, we have the same in the other direction:

3. If $\mathcal{S}_{B} \underset{r}{\stackrel{l}{\rightarrow}} \mathcal{S}_{B}^{\prime}$ and $r$ is the recipe of a rule in Env $\cup I F$, then there exists actions $l^{\prime} \in \mathcal{F}^{\sharp}$ and $\mathcal{S}_{A}^{\prime} \in \mathcal{G}^{\sharp}$ such that $\mathcal{S}_{A} \underset{r}{\stackrel{l^{\prime}}{\longrightarrow}} \mathcal{S}_{A}^{\prime}$, and $\left(\mathcal{S}_{A}^{\prime}, \mathcal{S}_{B}^{\prime}\right) \in \mathcal{R}$.

4. If $\mathcal{S}_{B} \underset{r}{\stackrel{l}{\rightarrow}} \mathcal{S}_{B}^{\prime}$ and $r$ is the recipe of a rule in $S_{B}$, then there exist recipes $r_{1}, \ldots, r_{n} \in \rho$ of rules in $S_{A}$, actions $l_{1}, \ldots, l_{n} \in \mathcal{F}^{\sharp}, n \geq 0$, and $\mathcal{S}_{A}^{\prime} \in \mathcal{G}^{\sharp}$ such that $\mathcal{S}_{A} \underset{r_{1}}{\stackrel{l_{1}}{\longrightarrow}}$ $\ldots \underset{r_{n}}{\stackrel{l_{n}}{\longrightarrow}} \mathcal{S}_{A}^{\prime}$, and $\left(\mathcal{S}_{A}^{\prime}, \mathcal{S}_{B}^{\prime}\right) \in \mathcal{R}$.

\subsection{Examples}

We now illustrate this definition on several examples.

EXAmple 5 (PAIRS). Consider the two systems and the environment from Example 4. In $S_{B} \cup I F \cup$ Env we have

$$
\begin{aligned}
& \emptyset \underset{B([\{m / x, n / y\}],[])}{\longrightarrow}\left\{\text { Out }_{\text {Sys }}((m, n))\right\} \\
& \underset{\text { OUT }([], z)}{\stackrel{O}{\longrightarrow}}\left\{\ln _{E n v}((m, n))\right\} \text {. }
\end{aligned}
$$

The only way for $S_{A} \cup I F \cup$ Env to simulate this would be

$$
\begin{aligned}
\emptyset & \underset{A([\{m / x\}],[])}{\longrightarrow}\left\{\text { Out }_{\text {Sys }}((m, m))\right\} \\
& \underset{O U T([], z)}{\longrightarrow}\left\{\operatorname{In}_{E n v}((m, m))\right\} .
\end{aligned}
$$

and potentially further transitions using rule A, adding more Out $_{S y s}((m, m))$ facts to the state. Note that there can only be one $\operatorname{In}_{E n v}((m, m))$-fact in the resulting state as the output transition can only be used once. This implies that for the resulting state $S$ we have $\left(\{S\},\left\{\operatorname{In}_{E n v}((m, n))\right\}\right) \in \mathcal{R}$. However we have

$$
S \underset{C\left([],\left[O U T_{1}([], z)\right]\right)}{\longrightarrow}\left\{\text { Out }_{E n v}(\text { true })\right\},
$$

but in state $\left\{\operatorname{In}_{E n v}((m, n))\right\}$ no transition with the same recipe is possible, hence $S_{A} \nsim_{E n v} S_{B}$.

This simple example illustrates that if the environment can do something on one side, but not on the other, then the two sides are distinguishable and therefore not observationally equivalent. The next example illustrates the importance of recipes in our definition of observational equivalence.

ExAmple 6. Consider the two systems from Example 4, but a different environment Env':

$$
E n v^{\prime}= \begin{cases} & E_{f s t}: \ln _{E n v}((x, y))-[\} \rightarrow M(x), \\ & E_{\text {snd }}: \operatorname{In}_{E n v}((x, y))-[] \rightarrow M(y), \\ & \left.E_{c m p}: M(x), M(x)-[] \rightarrow \text { Out }_{\text {Env }}(\text { true })\right\},\end{cases}
$$

where $M(\cdot)$ is a persistent fact. Intuitively we would expect that this environment can distinguish $S_{A}$ and $S_{B}$, as it can compare the first and second value of the tuple. We now try to apply the same reasoning as in Example 5. Consider

$$
\begin{aligned}
& \emptyset \underset{B([\{m / x, n / y\}],[])}{\longrightarrow}\left\{\text { Out }_{\text {Sys }}((m, n))\right\} \\
& \underset{\text { OUT([],z) }}{\stackrel{O}{\longrightarrow}}\left\{\operatorname{In}_{E n v}((m, n))\right\} \\
& \underset{E_{f s t}\left([],\left[O U T_{1}([], z)\right]\right)}{\longrightarrow}\{M(m)\} \\
& \underset{E_{\text {snd }}\left([],\left[O U T_{1}([], z)\right]\right)}{\stackrel{2}{\longrightarrow}}\{M(m), M(n)\} \text {. }
\end{aligned}
$$

In $S_{A} \cup I F \cup E n v^{\prime}$ this can be simulated as follows:

$$
\begin{aligned}
& \emptyset \underset{A([\{m / x\}],[])}{\longrightarrow}\left\{\text { Out }_{\text {Sys }}((m, m))\right\} \\
& \underset{O U T([], z)}{\stackrel{A}{\longrightarrow}}\left\{\operatorname{In}_{E n v}((m, m))\right\}
\end{aligned}
$$

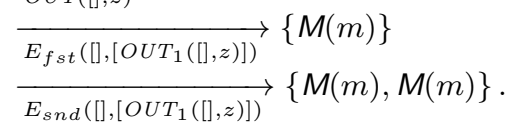

Moreover, we can compare the first and second value of the tuple with

$$
\{M(m), M(m)\} \underset{r_{1}}{\longrightarrow}\left\{M(m), M(m), \text { Out }_{\text {Env }}(\text { true })\right\},
$$

where

$$
\begin{array}{r}
r_{1}=E_{c m p}\left([],\left[E_{f s t, 1}\left([],\left[\operatorname{OUT}_{1}([], z)\right]\right),\right.\right. \\
\left.\left.E_{s n d, 1}\left([],\left[O U T_{1}([], z)\right]\right)\right]\right) .
\end{array}
$$

This transition cannot be matched by $S_{B} \cup I F \cup E n v^{\prime}$. Note however that the following transition is possible for $S_{B} \cup I F \cup$ $E n v^{\prime}$ :

$$
\{M(m), M(n)\} \underset{r_{2}}{\longrightarrow}\left\{M(m), M(n), \text { Out }_{\text {Env }}(\text { true })\right\},
$$

where

$$
\begin{array}{r}
r_{2}=E_{c m p}\left([],\left[E_{f s t, 1}\left([],\left[\operatorname{OUT}_{1}([], z)\right]\right),\right.\right. \\
\left.\left.E_{f s t, 1}\left([],\left[\operatorname{OUT}_{1}([], z)\right]\right)\right]\right) .
\end{array}
$$


The only difference between the two transition is the different recipe: instead of comparing the first and the second value of the tuple, we simply compared the first value to itself, and therefore they are not observational equivalent. This example shows that with a different environment the two systems are still distinguishable.

The next example shows how two different systems can behave in an equivalent way, and how equations can be used to model the equivalence of terms.

Example 7 (Coins). Consider a vending machine, in particular the part that returns coins as change when the money inserted was not fully spent. For simplicity we consider only $1 €$ and $2 €$ coins, represented by the functions one and two, and a constant null representing no coins. Now suppose we want to return $3 €$. The preferred solution would be to return two coins: $1 €$ and $2 €$. Yet returning three $1 €$ coins is also possible and, moreover, the order of the coins could be permuted.

Consider again two systems. The first system specifies the optimal behavior of returning just two coins:

$$
S_{A}=\left\{A:-[\} \text { Out }_{\text {Sys }}(\text { two }(\text { one }(\text { null })))\right\} .
$$

The second system, representing the actual implementation, may also return other combinations of coins. It is given by

$$
S_{B}=\left\{\begin{array}{l}
B_{1}:-\left[\rightarrow \text { Out }_{\text {Sys }}(\text { two }(\text { one }(\text { null }))),\right. \\
B_{2}:-\left[\rightarrow \text { Out }_{\text {Sys }}(\text { one }(\text { one }(\text { one }(\text { null })))),\right. \\
B_{3}:-\left[\rightarrow \rightarrow \text { Out }_{\text {Sys }}(\text { one }(\text { two }(\text { null })))\right.
\end{array}\right.
$$

We now define an environment that checks whether the implementation is correct with respect to the specification. Namely, the vending machine returns the same amount of money using the same coins returned in the same order:

$$
\begin{aligned}
& \text { Env }=\left\{\quad E_{\text {null }}:-[\} M(\text { null }),\right. \\
& E_{\text {one }}: M(x)-[\rightarrow M(\text { one }(x)) \text {, } \\
& E_{\text {two }}: M(x)-[] \rightarrow M(t w o(x)) \text {, } \\
& \left.E_{\text {check }}: M(x), \operatorname{In}_{\text {Env }}(x)-[\} \text { Out } \text { Env }(\text { true })\right\} \text {. }
\end{aligned}
$$

The environment's test works as follows. Using the first three rules, the environment can build any amount of money from the two kinds of coins. Then, using the $E_{\text {check }}$ rule, this can be compared to the system's output. Hence, for $S_{A} \approx_{E n v} S_{B}$ to hold, both systems must output the same amount of money using the same coins in the same order, otherwise $E_{\text {check }}$ is applicable only on one side. We have $S_{A} \approx_{E n v} S_{B}$ as the amount of money returned is the same, but the coins may differ: $S_{A}$ can only return two coins, while $S_{B}$ could also return three. More precisely, the environment could build the fact $M($ two(one(null))), and try to apply the rule $E_{\text {check. }}$. This would work for the system $S_{A}$ provided an output was made, but not necessarily for the system $S_{B}$ as the output could, for example, have been one (one $($ one $($ null $)))$.

Suppose that we add the equation two $(x)=$ one $($ one $(x))$, stating that a $2 €$ coin is equivalent to two $1 €$ coins. Then $S_{A} \approx_{E n v} S_{B}$ as the amount of money output by both machines is the same and two $($ one $($ null $))=$ one $($ two $($ null $))=$ one $($ one $($ one $(n u l l)))$. Hence the environment successfully checks whether both systems output the same amount of money, independent of the coins used.

Naturally we can also have other environments. Assuming no equations, consider the environment

$$
E n v^{\prime}=\left\{E_{c o m p}: \ln _{E n v}(x), \ln _{E n v}(x)-[\} \text { Out }_{E n v}(\operatorname{true})\right\} .
$$

This environment compares whether two system outputs are equal, which is not necessarily the case for $S_{B}$, but holds for $S_{A}$.

These examples illustrate the generality of our definition of observational equivalence: as it is parametrized by the environment, it can be instantiated in different ways depending on the application context. In protocol verification, this could for example be used to model different types of attackers. Note also that in other process algebras used for protocol verification, such as the applied $\pi$-calculus, the environment is typically implicitly defined and cannot be changed.

\section{PROVING OBSERVATIONAL EQUIVALENCE}

To automate proofs of observational equivalence we introduce the notion of a bi-system.

\subsection{Bi-Systems}

A bi-system is a multiset rewrite system where terms may be built using the special operator $\operatorname{diff}[\cdot, \cdot]$, indicating two possible instantiations corresponding to the left and right subterm. This use of diff operators was first introduced in PROVERIF [7] where bi-processes are handled in a similar fashion. Using diff-terms, one can specify two systems (left and right) with almost identical rules by one multiset rewriting system, where the only difference is how the diff-terms are instantiated. This simplifies the search for the simulation relation, as we can simply assume that each rule simulates itself, modulo the diff-terms. Nevertheless, this notion is expressive enough to specify many relevant security properties. These include all the examples mentioned in the introduction: our desired real-or-random test, privacy-related properties of voting and auctions, indistinguishability properties such as ciphertext indistinguishability, and authenticated key-exchange security. Moreover, as we show below, all examples from Section 3 can also be expressed this way.

For $S$ a bi-system, we can obtain its left instance $L(S)$ by replacing each term $\operatorname{diff}[M, N]$ in $S$ with $M$. Similarly, we can obtain $S$ 's right instance $R(S)$ by replacing each term $\operatorname{diff}[M, N]$ with $N$. These are both standard multiset rewrite systems. The goal of the algorithm we give is to prove that given a bi-system $S, L(S)$ and $R(S)$ are observationally equivalent.

We now revisit the Examples 3 and 7, starting with the tuple example.

EXAmple 8 (TUPles WITH DIFF). Using diff-terms we can define a single bi-system $S$ that combines $S_{A}$ and $S_{B}$ as

$$
S=\left\{A B: \multimap\left[\text { Out }_{\text {Sys }}((x, \operatorname{diff}[x, y]))\right\},\right.
$$

where $L(S)=S_{A}$ and $R(S)=S_{B}$, as in Example 3.

EXAMPle 9 (COINS WITH DIFF). We create a bi-system $S$ that merges $S_{A}$ and $S_{B}$. The left-hand side of each diffterm is the specification, while the right-hand side is the implementation:

$$
\begin{aligned}
& S=\left\{A B_{1}:-[\} \rightarrow \text { Out }_{\text {Sys }}(\operatorname{diff}[\text { two }(\text { one }(\text { null })),\right. \\
& A B_{2}:-[\} \operatorname{Out}_{\text {Sys }}(\operatorname{diff}[\text { two }(\text { one }(\text { null })), \\
& \text { one (one }(\text { one }(\text { null })))]) \\
& A B_{3}:-[\} \rightarrow \text { Out }_{\text {Sys }}(\operatorname{diff}[\text { two }(\text { one }(\text { null })), \\
& \text { one }(\text { two }(\text { null }))]) \text { \}. }
\end{aligned}
$$


Keeping the environment Env identical to Example 7 results in the bi-system $S$ not satisfying observational equivalence. But, if we add the equation two $(x)=$ one $($ one $(x))$, then $S$ satisfies observational equivalence.

\subsection{Dependency Graph Equivalence}

To simplify reasoning, our algorithm works with dependency graphs rather than with the labeled transition system. Dependency graphs are a data structure that formalize the entire structure of a system execution, including which facts originate from which rules, similar to recipes. We have several reasons for using dependency graphs. First, by capturing the entire system state, they are well-suited for automated analysis using constraint solving. Second, this representation is already implemented and supported in the TAMARIN prover $[28,26]$, which we extend. Finally, dependency graphs naturally give rise to an equivalence relation that implies observational equivalence; however, it is substantially simpler to verify.

Definition 2 (Dependency Graph). Let $E$ be an equational theory, $R$ be a set of labeled multiset rewriting protocol rules, and Env an environment. We say that the pair $d g=(I, D)$ is a dependency graph modulo $E$ for $R$ if $I \in_{E}$ ginsts $(R \cup I F \cup E n v)^{*}, D \in \mathcal{P}\left(\mathbb{N}^{2} \times \mathbb{N}^{2}\right)$, and dg satisfy the three conditions below. To state these conditions, we define the following notions. Let I be a sequence of rule instances whose indices, $i d x(I)$, represent the nodes of $d g$. We call $D$ the edges of $d g$ and write $(i, u) \longmapsto(j, v)$ for the edge $((i, u),(j, v))$. A conclusion of $d g$ is a pair $(i, u)$ such that $i$ $i s$ a node of $d g$ and $u \in i d x\left(\operatorname{concs}\left(I_{i}\right)\right)$. The corresponding conclusion fact is $\left(\operatorname{concs}\left(I_{i}\right)\right)_{u}$. A premise of $d g$ is a pair $(j, v)$ such that $j$ is a node of $d g$ and $v \in i d x\left(\operatorname{prems}\left(I_{j}\right)\right)$. The corresponding premise fact is $\left(\operatorname{prems}\left(I_{j}\right)\right)_{v}$. A conclusion or premise is linear if its fact is linear.

DG1 For every edge $(i, u) \longmapsto(j, v) \in D$, it holds that $i<j$ and the conclusion fact of $(i, u)$ is equal modulo $E$ to the premise fact of $(j, v)$.

DG2 Every premise of dg has exactly one incoming edge.

DG3 Every linear conclusion of dg has at most one outgoing edge.

We denote the set of all dependency graphs of $R$ modulo $E$ by $\operatorname{dgraphs}_{E}(R)$. Moreover, by state $(d g)$ we denote the set of all conclusion facts in $d g$ that are either persistent or (if they are linear) do not have an outgoing edge. This intuitively corresponds to the state of the LTS after all transitions in the dependency graph have been executed.

Figures 2 and 3 contain dependency graphs corresponding to evaluations based on Examples 6 and 7, respectively.

Using dependency graphs, we can define a stronger version of observational equivalence, which is used by our algorithm. For this, we define the dependency graphs of a rule, which intuitively corresponds to the set of all dependency graphs having the rule as root. Given a rule $r \in R \cup I F \cup E n v$, its dependency graphs dgraphs $_{E}(r)$ contain all dependency graphs where the last node, i.e., the node $(i, u)$ with maximal $i$, is an instance of the rule $r$. Moreover, by new diffvariables we mean the new variables of a rule that only appear in one of its two diff-variants, e.g., $y$ in the case of a rule Out $((x, \operatorname{diff}[x, y]))$, where $x$ and $y$ are new variables.

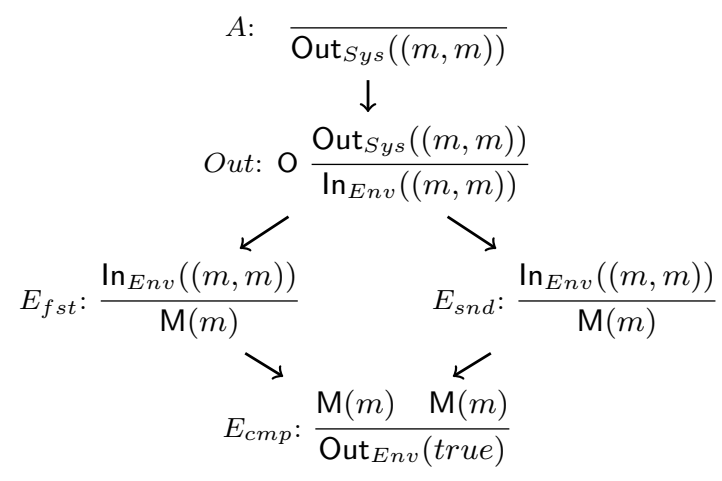

Figure 2: Dependency graph for Example 6

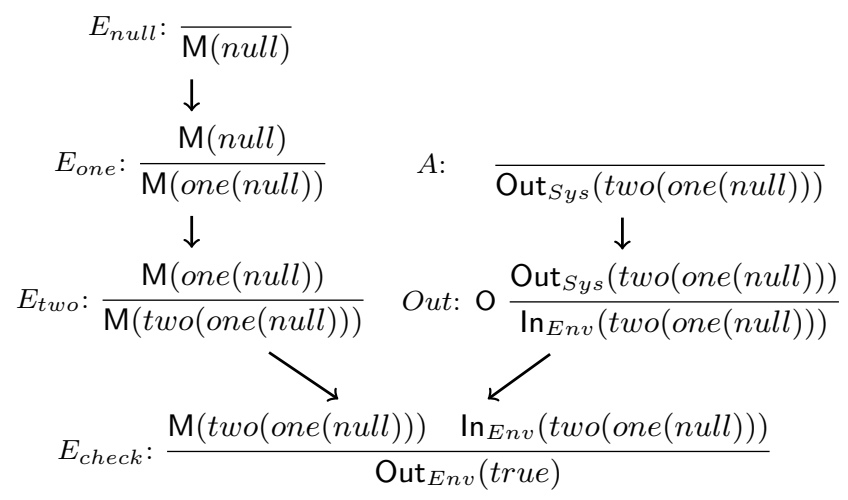

Figure 3: Dependency graph for Example 7

Finally, we define the mirrors of dependency graphs. Intuitively, given a dependency graph, its mirrors contain all dependency graphs on the other side of the bi-system with the same structure, notably the same edges and where the nodes are instances (potentially different due to the diffterms) of the same rules.

Suppose that for all dependency graphs of all rules, the set of its mirrors contains all "necessary" instances. We then know that - independently of the current state of the system - if a transition is enabled by a rule on one side, the same rule also enables a transition on the other side, implying observational equivalence. This is formalized in Definition 4 below as Dependency Graph Equivalence.

Definition 3 (Mirroring Dependency Graphs). Let $S$ be a protocol bi-system and Env be an environment. Consider the multiset rewrite systems $L=L(S) \cup I F \cup E n v$ and $R=R(S) \cup I F \cup E n v$.

Let $d g_{L}=\left(I_{L}, D_{L}\right) \in$ dgraphs $(L)$ be a dependency graph. We denote by mirrors $\left(d g_{L}\right)$ the set of all dependency graphs $d g_{R}=\left(I_{R}, D_{R}\right) \in$ dgraphs $(R)$, such that $D_{R}=D_{L},\left|I_{L}\right|=$ $\left|I_{R}\right|, i d x\left(I_{L}\right)=i d x\left(I_{R}\right)$ and for all $i \in i d x\left(I_{L}\right)$ the ground rule instances $\left(I_{L}\right)_{i}$ and $\left(I_{R}\right)_{i}$ are ground instances of the same rules, i.e., rules with the same identifier, where new variables of rules keep their instantiation.

The set of mirrors of a dependency graph $d g_{R}=\left(I_{R}, D_{R}\right) \in$ dgraphs $(R)$, denoted by mirrors $\left(d g_{R}\right)$, is defined analogously, replacing $R$ by $L$ uniformly in the above definition.

Using these notions, we now define dependency graph equiv- 
alence. Intuitively, this definition captures that for all dependency graphs on one side of the bi-system, there is a mirroring dependency graph on the other side that respects its instantiations of new diff-variables.

Definition 4 (Dependency Graph Equivalence). Let $S$ be a bi-system. Consider the multiset rewrite systems $L=L(S) \cup I F \cup E n v$ and $R=R(S) \cup I F \cup E n v$. We say that $L$ and $R$ are dependency graph equivalent, written $L(S) \sim_{D G, E n v} R(S)$, if for all dependency graphs $d g$ of rules $r \in L \cup R$, the set mirrors $(d g)$ is non-empty and contains dependency graphs for all possible instantiations of new diffvariables.

Note that this definition requires that if, for example, there are new variables in the rules $R$ not appearing in the rules from $L$ used in $d g$, then mirrors $(d g)$ must contain instances for all possible instantiations of these variables. For instance, in the case of a rule producing a conclusion Out $((x, \operatorname{diff}[x, y]))$, then for all possible instantiations of $y$, an instance must be in mirrors $(d g)$.

It turns out that dependency graph equivalence is a sufficient (but not necessary) criterion for observational equivalence. Intuitively, dependency graph equivalence verifies that the left-hand side instance of a rule can always be simulated by its right-hand side, and vice versa.

THEOREM 1. Let $S$ be a bi-system. If $L(S) \sim_{D G, E n v}$ $R(S)$ then $L(S) \approx_{E n v} R(S)$.

Proof. Consider the multiset rewrite systems $L=L(S) \cup$ $I F \cup E n v$ and $R=R(S) \cup I F \cup E n v$, and the relation $\mathcal{R}$ :

$$
\begin{array}{cll}
\mathcal{R}=\left\{\left(\mathcal{S}_{A}, \mathcal{S}_{B}\right) \mid\right. & \mathcal{S}_{A}=\operatorname{state}\left(d g_{L}\right), \mathcal{S}_{B}=\operatorname{state}\left(d g_{R}\right), \\
& \left.d g_{R} \in \operatorname{mirrors}\left(d g_{L}\right), d g_{L} \in \operatorname{dgraphs}(L)\right\} \\
\cup\left\{\left(\mathcal{S}_{A}, \mathcal{S}_{B}\right) \mid\right. & \mathcal{S}_{A}=\operatorname{state}\left(d g_{L}\right), \mathcal{S}_{B}=\operatorname{state}\left(d g_{R}\right), \\
& \left.d g_{L} \in \operatorname{mirrors}\left(d g_{R}\right), d g_{R} \in \operatorname{dgraphs}(R)\right\} .
\end{array}
$$

First note that $(\emptyset, \emptyset) \in \mathcal{R}$. We now show that $\mathcal{R}$ is an observational equivalence relation as defined in Definition 1. For this, we must show that for all states $\left(\mathcal{S}_{A}, \mathcal{S}_{B}\right) \in \mathcal{R}$ we have:

1. If $\mathcal{S}_{A} \underset{r}{\stackrel{l}{\rightarrow}} \mathcal{S}_{A}^{\prime}$ and $r$ is the recipe of a rule in $I F \cup E n v$, then there exists actions $l^{\prime} \in \mathcal{F}^{\sharp}$ and $\mathcal{S}_{B}^{\prime} \in \mathcal{G}^{\sharp}$ such that $\mathcal{S}_{B} \underset{r}{\stackrel{l^{\prime}}{\rightarrow}} \mathcal{S}_{B}^{\prime}$, and $\left(\mathcal{S}_{A}^{\prime}, \mathcal{S}_{B}^{\prime}\right) \in \mathcal{R}$.

2. If $\mathcal{S}_{A} \underset{r}{\stackrel{l}{\rightarrow}} \mathcal{S}_{A}^{\prime}$ and $r$ is the recipe of a rule in $S_{A}$, then there exist recipes $r_{1}, \ldots, r_{n} \in \rho$ of rules in $S_{B}$, actions $l_{1}, \ldots, l_{n} \in \mathcal{F}^{\sharp}, n \geq 0$, and $\mathcal{S}_{B}^{\prime} \in \mathcal{G}^{\sharp}$ such that $\mathcal{S}_{B} \underset{r_{1}}{\stackrel{l_{1}}{\longrightarrow}}$ $\ldots \underset{r_{n}}{\stackrel{l_{n}}{\longrightarrow}} \mathcal{S}_{B}^{\prime}$, and $\left(\mathcal{S}_{A}^{\prime}, \mathcal{S}_{B}^{\prime}\right) \in \mathcal{R}$.

We distinguish the following cases:

1. Assume $\left(\mathcal{S}_{A}, \mathcal{S}_{B}\right) \in \mathcal{R}, \mathcal{S}_{A} \underset{r}{\stackrel{l}{\rightarrow}} \mathcal{S}_{A}^{\prime}$ for a rule instance $r i$, and $r$ is the recipe of a rule in $I F \cup E n v$. Then, by the definition of $\mathcal{R}$, there is a dependency graph $d g_{L} \in \operatorname{dgraphs}(L)$ with $\mathcal{S}_{A}=\operatorname{state}\left(d g_{L}\right)$, and a dependency graph $d g_{R} \in \operatorname{dgraphs}(R)$ with $\mathcal{S}_{B}=\operatorname{state}\left(d g_{R}\right)$. Since the transition $\mathcal{S}_{A} \underset{r}{\stackrel{l}{\rightarrow}} \mathcal{S}_{A}^{\prime}$ is possible in $\mathcal{S}_{A}, d g_{L}$ can be extended to $d g_{L}^{\prime}$ with the rule instance $r i$ corresponding to this transition, and $\operatorname{state}\left(d g_{L}^{\prime}\right)=\mathcal{S}_{A}^{\prime}$.
Then $d g_{L}^{\prime} \in d \operatorname{dgraph}(L)$, and by $L(S) \sim_{D G, E n v} R(S)$ we have that for all possible instantiations of new diff variables, the corresponding dependency graph $d g_{R}^{\prime} \in$ $d g r a p h s(R)$. By the definition of $\mathcal{R}$, in $d g_{R}$ the instantiations of the new variables (including the new diff-variables) correspond to the instantiations of some $d g_{R}^{\prime} \in \operatorname{mirrors}\left(d g_{L}^{\prime}\right)$. Then, by the construction of mirrors $\left(d g_{L}^{\prime}\right), d g_{R}^{\prime}$ is identical to $d g_{R}$ except for the last rule instance $r i^{\prime}$. Moreover, by the construction of mirrors $\left(d g_{L}^{\prime}\right), r i^{\prime}$ is an instance of the rule with the same identifier. Since the dependency graph $d g_{R}^{\prime}$ has the same structure $D$ as $d g_{L}^{\prime}$ and all rules in $I F \cup E n v$ have no new diff-variables, there exists a transition $\mathcal{S}_{B} \stackrel{l^{\prime}}{\rightarrow} \mathcal{S}_{B}^{\prime}$ with the same recipe as ri. Moreover, $\left(\mathcal{S}_{A}^{\prime}, \mathcal{S}_{B}^{\prime}\right) \in \mathcal{R}$ since there are mirroring dependency graphs for $\mathcal{S}_{A}^{\prime}$ and $\mathcal{S}_{B}^{\prime}$.

The symmetric case is analogous.

2. Alternatively, assume $\left(\mathcal{S}_{A}, \mathcal{S}_{B}\right) \in \mathcal{R}, \mathcal{S}_{A} \stackrel{l}{\rightarrow} \mathcal{S}_{A}^{\prime}$, and $r$ is the recipe of a rule in $L(S)$. Then, by the definition of $\mathcal{R}$, there is a dependency graph $d g_{L} \in \operatorname{dgraphs}(L)$ with $\mathcal{S}_{A}=\operatorname{state}\left(d g_{L}\right)$. Since in this state the transition $\mathcal{S}_{A} \underset{r}{\stackrel{l}{\rightarrow}} \mathcal{S}_{A}^{\prime}$ is possible, $d g_{L}$ can be extended to $d g_{L}^{\prime}$ with the rule instance $r i$ corresponding to this transition, and state $\left(d g_{L}^{\prime}\right)=\mathcal{S}_{A}^{\prime}$. Then $d g_{L}^{\prime} \in \operatorname{dgraphs}(L)$, and by $L(S) \sim_{D G, E n v} R(S)$ we have that for all possible instantiations of new diff variables, the corresponding dependency graph $d g_{R}^{\prime} \in \operatorname{dgraphs}(R)$. By the definition of $\mathcal{R}$, there is a dependency graph $d g_{R} \in \operatorname{dgraphs}(R)$ with $\mathcal{S}_{B}=\operatorname{state}\left(d g_{R}\right)$, where the instantiations of the new variables (including the new diff-variables) correspond to the instantiations of some $d g_{R}^{\prime} \in \operatorname{mirrors}\left(d g_{L}^{\prime}\right)$. Then, by the construction of mirrors $\left(d g_{L}^{\prime}\right)$, this graph $d g_{R}^{\prime}$ is identical to $d g_{R}$ except for the last rule instance. By assumption, $r i$ was an instance of a rule in $L(S)$. Therefore, by the construction of mirrors $\left(d g_{L}^{\prime}\right)$, the last rule instance $r i^{\prime}$ in $d g_{R}^{\prime}$ is an instance of the rule with the same identifier. Hence there exists a transition $\mathcal{S}_{B} \underset{r^{\prime}}{\stackrel{l^{\prime}}{\longrightarrow}} \mathcal{S}_{B}^{\prime}$. Moreover, $\left(\mathcal{S}_{A}^{\prime}, \mathcal{S}_{B}^{\prime}\right) \in \mathcal{R}$ since there are mirroring dependency graphs for $\mathcal{S}_{A}^{\prime}$ and $\mathcal{S}_{B}^{\prime}$.

Again, the symmetric case is analogous.

As shown in [28], we can use constraint solving to find (restricted) normal dependency graphs; for a detailed discussion about the constraint solving procedure used and the link to restricted normal dependency graphs, see the extended version of this paper [1]. This provides the basis for our algorithm, depicted in Figure 4, which determines whether $L(S) \sim_{D G} R(S)$ holds. For each rule $r$ in $L(S)$, $R(S)$, and the environment, the algorithm finds all corresponding normal dependency graphs with $r$ as a root using constraint solving. For each of these dependency graphs, it then checks whether the set of mirrors contains all instances required for normal dependency graph equivalence. If this holds, it reports that verification is successful; otherwise it returns the dependency graph that lacks a mirroring instance as a counterexample. Note that these instances are counterexamples to dependency graph equivalence, but not necessarily to observational equivalence because of the approximation requiring that each rule is simulated by itself. 
1: function $\operatorname{VERIFY}(\mathrm{S})$

2: $\quad R U \leftarrow L(S) \cup R(S) \cup I F \cup E n v$

3: $\quad$ while $R U \neq \emptyset$ do

4: $\quad$ choose $r \in R U, R U \leftarrow(R U \backslash\{r\})$

5: $\quad$ compute $D G \leftarrow \operatorname{dgraphs}(r)$ by constraint solving

6: $\quad$ if $\exists d g \in D G$ s.t. mirrors $(d g)$ lacks ground instances

7: then return "potential attack found: ", $d g$

8: return "verification successful"

Figure 4: Pseudocode of our verification algorithm.

This is due to the undecidability of the initial problem, and all related tools $[9,11,8,7,29]$ also have this limitation.

We now explain how we adapt and use this algorithm in TAMARIN. We provide examples of its use in Section 5 .

\subsection{Tamarin}

The TAMARIN prover $[28,26]$ is a security protocol verification tool that supports both the falsification and unbounded verification of security protocols specified as multiset rewriting systems with respect to trace-based properties.

In TAMARIN, a security protocol $P$ 's executions are modeled by its set of traces, defined as the concatenation of the sets of action labels at each step. A trace is a sequence of facts denoting the sequence of actions taken during a protocol's execution. The trace of an execution

$$
S_{0},\left(l_{1} \underset{r e c_{1}}{\stackrel{a_{1}}{\longrightarrow}} r_{1}\right), S_{1}, \ldots, S_{k-1},\left(l_{k} \underset{r e c_{k}}{\stackrel{a_{k}}{\longrightarrow}} r_{k}\right), S_{k}
$$

is the sequence of the multisets of its action labels $\left[a_{1}, \ldots, a_{k}\right]$.

We now briefly recall the TAMARIN prover's adversary message derivation rules $M D$. To define the protocol and adversary rules, we assume that $\Sigma_{\text {Fact }}$ includes the persistent fact symbol $\mathrm{K}$ modeling messages known to the adversary, the linear fact symbol Out modeling messages sent by the protocol, and the linear fact symbol In modeling messages sent by the adversary. The adversary's message deduction capabilities are captured by the following set of rules.

$$
\begin{aligned}
& M D=\{\operatorname{Out}(t)-[] \rightarrow \mathrm{K}(t), \mathrm{K}(t)-[\mathrm{K}(t)] \rightarrow \ln (t), \\
&\operatorname{Fr}(x: f r)-[\} \mathrm{K}(x: f r),[]-[] \rightarrow \mathrm{K}(x: p u b)\} \\
& \cup\left\{\mathrm{K}\left(t_{1}\right), \ldots, \mathrm{K}\left(t_{n}\right)-[] \rightarrow \mathrm{K}\left(f\left(t_{1}, \ldots, t_{n}\right)\right) \mid f \in \Sigma_{\text {Fun }}^{n}\right\}
\end{aligned}
$$

The adversary learns all messages that are sent and it can send any message it knows (i.e., it learns or can derive) to the protocol. It can generate fresh values and it knows all public values. Additionally, the adversary can apply all operators to terms it knows. When using an equational theory, each of the equations gives rise to a deconstruction rule that lets the intruder derive the result. For example for symmetric encryption and decryption, with the equation $\operatorname{sdec}(\operatorname{senc}(m, k), k)=m$, TAMARIN automatically generates the rule $\mathrm{K}(\operatorname{senc}(m, k)), \mathrm{K}(k)-[\} \rightarrow \mathrm{K}(m)$, which the adversary uses to decrypt messages.

We also add one new adversary deduction rule to $M D$, which we call IEquality, which allows the adversary to compare two values for equality:

$$
\text { IEquality : } \mathrm{K}^{\downarrow}(x), \mathrm{K}^{\uparrow}(x)-[] \rightarrow .
$$

The use of $\mathrm{K}^{\downarrow}$ and $\mathrm{K}^{\uparrow}$ in this rule restricts how the adversary can derive the terms. ${ }^{1}$ Here, this annotation is crucial

\footnotetext{
${ }^{1}$ In all other rules $\mathrm{K}$ is actually also either $\mathrm{K}^{\downarrow}$ or $\mathrm{K}^{\uparrow}$. How-
} ever, this distinction is required only for automation (namely as we want to compare two terms that are derived separately. Moreover, it also prevents immediate non-termination: otherwise, once two values are successfully compared, one could compare their hashes, followed by their hashes, etc.

The IEquality rule is applicable whenever one side of a bi-system can construct the same value twice (but in different ways), that is it has dependency graphs as premises for both instances of $x$. Note that the other bi-system side trivially has the mirroring dependency graph if an output is compared with itself (same dependency graph twice), for example. But, if on one side the adversary can decrypt a message and compare it with the content, while on the other side that is not possible, then the IEquality rule will expose this. The analysis of the IEquality rule presented in Examples 10 and 11 illustrates this point in more detail.

For the details of our modification to the TAMARIN prover we refer the reader to the extended version of this paper [1]. There we show how our model can be instantiated for TAMARIN, and present an implementation of the above algorithm for verifying observational equivalence with TAMARIN. Note that just like the algorithm outlined in Figure 4 (line 4), TAMARIN carries out a rule-by-rule analysis.

The main challenges in implementing our algorithm in the TAMARIN prover relate to limiting the size of the state-space, which requires fine-tuning TAMARIN's internal heuristic. To aid termination, we restrict traces to normal forms as much as possible. Moreover, compared to the original TAMARIN prover, we needed to remove some of its normal form conditions because they are sound for trace properties, but not for observational equivalence. One such example is the normal form condition prohibiting repeated adversarial derivation of a term. However, equality comparison with previous values must be possible, e.g., to test whether the output equals the input in the protocol $\ln (x), \operatorname{Fr}(y)-[\} \rightarrow \operatorname{Out}(\operatorname{diff}(x, y))$.

\section{CASE STUDIES}

We now present four case studies. We first apply TAMARIN to two standard examples that have been analyzed using other tools. Afterwards we present two examples: one that is outside the scope of previous work and one that verifies a practical RFID protocol that had previously received manual analysis only. Note that all proofs are constructed in our tool completely automatically, with the exception of the attack on TPM_Envelope. For this protocol, interaction was limited to human input at a few key choice points and the remainder was automated. We provide a file containing the steps necessary to derive the identified attack for TPM_Envelope. For the other protocols, we just give their specification as TAMARIN's built-in strategy finds the proofs. All example files can be loaded into our extension of TAMARIN and are available at [1], together with TAMARIN. From now on, we use TAMARIN to refer to our extension, instead of the original TAMARIN.

\subsection{Motivating examples}

We start with two well-known examples from [7, 10]. For each example, we explain how TAMARIN determines observational equivalence using the algorithm VERIFY, presented in Figure 4.

state-space reduction and improving termination) so we have omitted it for ease of presentation. Full details can be found in the extended version [1]. 
Example 10 (Probabilistic Encryption). Consider the equational theory:

$$
\operatorname{pdec}(\operatorname{penc}(m, p k(k), r), k) \simeq m .
$$

This equation gives rise to the following decryption rule for probabilistic encryption for the adversary, which is automatically generated by TAMARIN:

$$
\text { Dpenc : } \mathrm{K}(\operatorname{penc}(m, p k(k), r)), \mathrm{K}(k)-[] \rightarrow \mathrm{K}(m) .
$$

We now express, as a bi-system, that a probabilistic encryption cannot be distinguished from a random value:

$$
S=\left\{\begin{array}{ll}
\text { GEN : } & \operatorname{Fr}(k)-[\} \rightarrow \operatorname{Key}(k), \operatorname{Out}(p k(k)) \\
E N C: & \operatorname{Key}(k), \operatorname{Fr}\left(r_{1}\right), \operatorname{Fr}\left(r_{2}\right), \operatorname{In}(x)-[] \rightarrow \\
& \operatorname{Out}\left(\operatorname{diff}\left[r_{1}, \operatorname{penc}\left(x, p k(k), r_{2}\right)\right]\right)
\end{array}\right\} .
$$

We summarize below how TAMARIN automatically proves this property. The algorithm VERIFY (line 2) first constructs the set $R U$ of rules to be analyzed,

$$
R U=\left\{\begin{array}{l}
L(G E N), R(G E N), L(E N C), R(E N C), \\
\text { FrESH } \left._{S y s}, \text { FrESH }_{E n v}, \text { IEquality, Dpenc }\right\},
\end{array}\right.
$$

together with the remaining rules in $I F$ and Env. Recall that $L$ (name) represents the rule name instantiated with the left side of the diff-term, and likewise for $R$ (name) with the right side. Then VERIFY iterates over all rules (lines 3-4) until either an attack is found (line 7) or all rules have been checked and the verification is complete (line 8), which happens in this example.

We now describe, for each rule, how VERIFY processes it. VERIFY first generates dependency graphs with the rule as the root (line 5). Afterwards, for each resulting dependency graph, it looks for a mirror (line 6) that contains all instances required by the definition of normal dependency graph equivalence. In this example, it always finds a mirror and verification therefore succeeds. Due to space and readability constraints, we present the left-diff instantiation and right-diff instantiation of each rule together, even though TAMARIN analyzes them independently. Due to space constraints, we also do not explicitly present the dependency graphs; however, we do explain how they are mirrored in each case so that the verification succeeds.

- As rule $G E N$ does not contain a diff-term, the left diff-instantiation of this rule is identical to the right diff-instantiation. The rule has only a single fresh fact as its premise and thus any dependency graph with this rule at its root contains only those two rule instances and is trivially mirrored by itself.

- The rule $E N C$ has the same premises in the left- and right-hand side system and is therefore identical for the purpose of dependency graph computation with the $E N C$ rule as root. (Note that outputs will be considered using the equality rule below.) The two fresh premises will result in identical dependency graphs, while the key and message reception input are independent. Hence both of them will have identical dependency graphs as premises, and the resulting dependency graphs are identical (up to the outputs) and therefore mirror each other.

- The fresh rules FRESH $_{S y s}$ and FRESHEnv have no premises. Hence the dependency graphs with them as root are just their instances, which mirror each other in the left- and right-hand system.
- For an equality rule instance of IEquality as the root of a dependency graph, the two premises are the same instance of a variable $x$. If both of the premises are adversary generated, then the resulting dependency graphs are the same in the left- and right-hand system, and thus will mirror themselves trivially. Alternatively, if one of the premises uses the output of an instance of either the $E N C$ rule or the $G E N$ rule, then there is no dependency graph with a matching second premise. This is because all system outputs, $p k(k)$ for $G E N$ and $r 1$ or $p e n c(x, p k(k), r 2)$ for $E N C$, contain a fresh value, $k, r 1$, respectively $r 2$, that is never available to the intruder. As this will never allow a complete dependency graph to be derived, no mirroring dependency graph is needed.

- For the decryption rule generated for the probabilistic encryption, this rule is never applicable on either side as the adversary never receives the keys needed for decrypting system generated encryptions. As there is no dependency graph, no mirroring one is needed. (One might mistakenly think that this rule might apply to intruder-generated terms. However, this is not the case due to the restrictions on how the adversary may combine its knowledge ( $\mathrm{K}^{\downarrow}$ vs $\mathrm{K}^{\uparrow}$ ) and, in any case, both sides would use the same dependency graphs as premise, so the result would be the same.)

- For all other adversary rules, it is obvious that they result in identical dependency graphs on both sides. More precisely: construction rules have adversary knowledge input and thus the same dependency graphs as premises. For the deconstruction rules, the only relevant one is the previous decryption rule, as that is the only one that can use information coming out of the system; all other rules can only be used on adversarygenerated terms and thus have the same dependency graphs as premises.

This completes our summary of TAMARIN's verification of observational equivalence for this example. TAMARIN automatically constructs the proof in under 0.2 seconds.

Our next example is Decisional Diffie-Hellman as discussed in [7, Example 2]. TAMARIN verifies the expected result that the adversary cannot distinguish a Diffie-Hellman tuple from a random tuple. Note that in contrast to [7], which uses an equational theory restricted just to the commutativity of two exponents, TAMARIN supports a substantially more comprehensive model of Diffie-Hellman exponentiation.

Example 11 (Decisional Diffie-Hellman). We use the equational theory for Diffie-Hellman exponentiation with an abelian group of exponents as provided by TAMARIN. Hence no additional adversary rules are needed.

We consider a single rule, which outputs the two half-keys and challenges the adversary to distinguish the actual key from an unrelated randomly generated key:

$$
\begin{array}{ll}
\text { GEN : } & \operatorname{Fr}\left(a_{1}\right), \operatorname{Fr}\left(a_{2}\right), \operatorname{Fr}\left(a_{3}\right)-[\} \rightarrow \\
& \operatorname{Out}\left(b^{a_{1}}, b^{a_{2}}, \operatorname{diff}\left[b^{a_{3}},\left(b^{a_{1}}\right)^{a_{2}}\right]\right) .
\end{array}
$$

Using the VERIFY algorithm, TAMARIN collects the rules

$$
R U=\left\{\begin{array}{l}
L(G E N), R(G E N), \\
\text { FrESH } \left._{\text {Sys }}, \text { FrESH }_{\text {Env }}, \text { IEquality }\right\},
\end{array}\right.
$$


together with the remaining rules in $I F$ and Env. We consider the processing of these rules below, where we again combine the treatment of left-diff instantiations and rightdiff instantiations of system rules to improve readability.

- The rule $G E N$ has only fresh facts as premise and thus any dependency graph with this rule at its root contains at most four rule instances, three of fresh rules and one of $G E N$ itself. Thus, it is mirrored trivially by itself. The mirror is actually identical (up to the output).

- The fresh rules Fresh Sys $_{\text {and }}$ Freshenv do not have premises. Hence the dependency graphs with them as roots are just their instances, which mirror each other on the left- and right-hand side.

- For an equality rule instance of IEquality as the root of a dependency graph, the two premises are the same instance of a variable $x$. If both of the premises are adversary generated, then the resulting dependency graphs are the same in the left- and right-hand system, and thus will mirror themselves trivially. Alternatively, if one of the premises uses the output of an instance of the $G E N$ rule, then there is no dependency graph with a matching second premise, except the one using the same source twice. This is because all of the system outputs cannot be related in meaningful fashion within the Diffie-Hellman exponentiation theory as it does not allow the extraction of exponents, which corresponds to computing discrete logs. As this will never allow a complete dependency graph to be derived, no mirroring dependency graph is needed. In the case of the same source being used twice, i.e., a value being compared with itself, the same premise dependency graphs work for both systems.

Additionally, note that multiple instances of the $G E N$ rule are entirely unrelated and do not provide any advantage for the adversary. TAMARIN analyzes this and computes all possible variants, determining that no combination is useful.

- For all other adversary rules, it is obvious that they result in identical dependency graphs on both sides. Namely, the construction rules have adversary knowledge input and thus the same dependency graphs as premises

The VERIFY algorithm therefore returns that verification is successful. TAMARIN verifies this, completely automatically, in 15.2 seconds.

This concludes our two motivating examples. They were small enough that we could give relatively detailed descriptions of VERIFY's workings. For subsequent examples, we will be more concise. Readers interested in the full gory details may generate them themselves by using TAMARIN and running the files for each case study.

\subsection{Feldhofer's RFID protocol}

The RFID protocol due to Feldhofer et al. [20] is of practical interest as it can be implemented with relatively few logic gates using AES encryption and hence it fits well with the requirements of current RFID chips. We use the description from [30] as the basis of our model, which we present in

$$
\begin{array}{ll}
R \rightarrow T: & n r \\
T \rightarrow R: & \{|n r, n t|\}_{k(R, T)} \\
R \rightarrow T: & \{|n t, n r|\}_{k(R, T)}
\end{array}
$$

\section{Figure 5: RFID protocol}

Figure 5 using Alice\&Bob notation. The protocol is between a reader $R$ and a tag $T$ that share a key $k(R, T)$. Note that $\{|\ldots|\}_{k}$ denotes symmetric encryption in this example.

In the first message, the reader sends a random nonce to the tag. In the second message, the tag sends back that nonce and one of its own choosing, encrypted with the shared key. In the third message, the reader responds with the same nonces in reverse order, also encrypted.

The desired security property for this protocol is privacy for the tags. Namely, if at least two tags share a key with a reader (and in practice, there will be many such tags), then the adversary cannot determine which tag is actually communicating with the reader. TAMARIN verifies this in under 1.6 seconds.

\subsection{TPM_Envelope protocol}

We first briefly explain the key part of this protocol [15] and afterwards present the TAMARIN rules along with further details. A stateful Trusted Platform Module (TPM) creates a one-use public/private key pair, and publishes the public key. A participant Alice then encrypts a nonce (the secret) with the public key (creating an envelope), which she sends to a participant Bob. Bob then either requests from the TPM the envelope's content, learning the secret, or requests a TPM-signed certificate stating that he did not ask for the content. In both cases, the TPM complies with the request and changes its state in such a way that it can afterwards only comply with repetitions of the first request, but never with the other request. This is where mutable state is crucial, i.e., the original capability of issuing either the certificate or the secret is revoked once the choice is made. The trace-based secrecy property verified in [15] states that the adversary may learn either the certificate or the secret, but not both.

We investigated whether this protocol additionally satisfies the real-or-random property for the secret. We therefore added a real-or-random challenge to the end of Alice's protocol execution, which sends out either the real secret or a random value. TAMARIN fails to prove this property and instead returns a simple attack, provided the encryption used is deterministic. The attack is as follows: the adversary (impersonating Bob) asks for the proof of never having received the secret from the TPM, and thus he must be unable to learn the secret. But, he can still distinguish whether the real-or-random challenge emits the real secret or a random value. He does this using the previously published public key, and encrypting the emitted value with it. Afterwards, he compares the resulting encryption to the envelope, and he learns that it is the real secret if it matches the envelope and a random value otherwise.

Inspecting this attack it is easy to see that it fails when probabilistic encryption is used instead. The adversary can still encrypt the emitted value with the public key, but the equality check against the envelope will always fail because the added randomness is different in the envelope and the adversary-generated comparison encryption.

In Figure 6 we present the rules used to model the pro- 
tocol TPM_Envelope in TAMARIN. Note that we omit here some details, which can be found in the model file included at [1]. First we explain the rules concerning the TPM's platform configuration registers (PCR). The Init rule initializes the PCR to the initial string ' $p c r 0^{\prime}$, and generates the fresh authentication identification key aik stored in the persistent AlK fact and sends out the public key $p k(a i k)$. This models a long-term key for the TPM. The extension rule Extend allows any PCR to be extended to the hash of the concatenation of its previous value and an input. This is used when a client later either extends the PCR with 'deny' or 'obtain'. This changes the TPM's state to allow creation of a certificate that the envelope was not opened ('deny'), or respectively opens the envelope ('obtain'). Rule CertK certifies a public key for which the TPM has stored the associated private key in the persistent key table fact KT with a particular lock. Locks are PCR values and the TPM will only extract the private key when the PCR value matches this lock. In the rule Quote, the current PCR value is sent out authentically, signed with the TPM's long-term key. The TPM's last rule is Unbind. It takes an envelope as input, and if the public key used to encrypt the envelope matches the private key in the key table, where additionally the lock in the key table matches the current PCR value, then the message in the envelope is decrypted and sent out.

The participant Alice requests an envelope key in her first rule $A 1$ by extending the PCR with a nonce $n$ of her choice. In rule $A 2$, she creates the secret to be put in the envelope encryption and checks that the TPM certifies that the key can only be obtained if the PCR state is extended with 'obtain' and then she uses the certified public key to encrypt her secret. Alice then publishes the envelope while keeping state in A2 for her next rule and in A2ror for the real-orrandom challenge. Rule $A 3$ uses the state in A2 to check that the TPM's PCR was extended with 'deny' (which means it has not yet, and can now no longer, decrypt the envelope) and then notes the action Denied. We are only interested in traces where the adversary can show this certificate. The rule CLKey is used with 'obtain' as the lock input to add a new private key to the TPM's key table that is used in rule $A 2$. It can of course be used with other inputs, but the resulting keys are not interesting to us. Now the key can only be extracted with a PCR extended with 'obtain', and thus the certificate with 'deny' is unavailable. The last rule is the ROR rule; this either outputs the real secret or a random value.

The TAMARIN prover finds the attack described above for the observational equivalence of the TPM_Envelope protocol. Note that this is a stronger property than trace-based secrecy, which had been verified by [15], so the two results are compatible. We therefore conclude that this protocol should only be used with probabilistic encryption, and not with deterministic encryption.

This example illustrates TAMARIN's handling of mutable state, which other tools cannot handle. It also illustrates the difference between trace-based properties and observational equivalence-based properties.

\section{RELATED WORK AND CONCLUSION}

We have shown how to take the well-established modeling formalism of multiset rewriting and extend it with a novel definition of observational equivalence. The result is well-suited for the verification of cryptographic protocols,

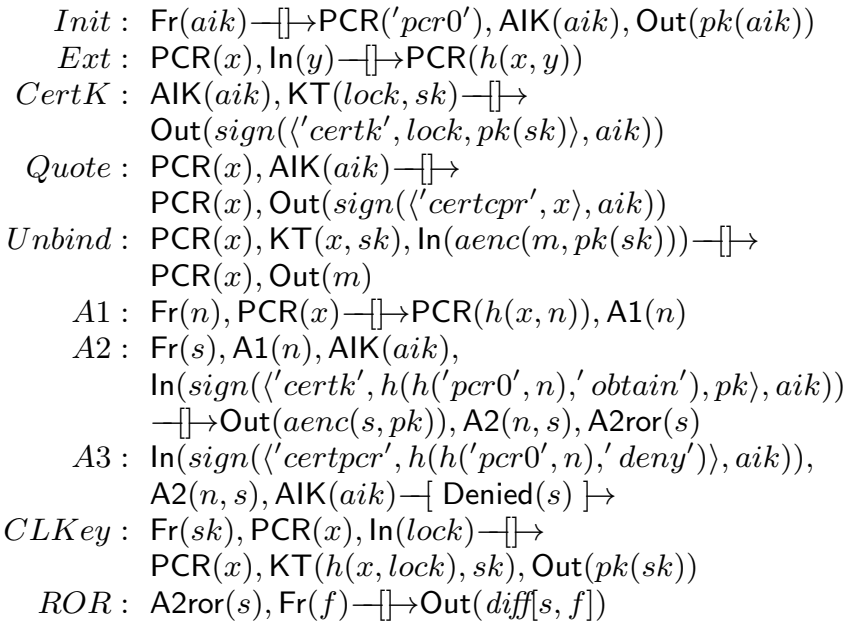

Figure 6: Rule set modeling the TPM_envelope

as well as other applications. Based on this, we have implemented an algorithm to prove observational equivalence for protocols specified in multiset rewriting and demonstrated its effectiveness on a number of case studies. Combining TAMARIN's constraint solving with the bi-system notion results in our approach's high degree of automation.

Our equivalence notion has similarities with other notions of observational equivalence considered in the literature, including trace equivalence [11], bisimulation [2], and notions based on contexts $[2,11,7]$. However, multiset rewriting and our observational equivalence definition are more flexible than the previous approaches as we can choose the environment as well as the underlying equational theory. As illustrated in Example 5 in Section 3, this can, for example, be used to model different types of attackers. In process algebras used for protocol verification, like the applied $\pi$-calculus, the environment is implicitly defined and cannot be changed. Moreover, we support mutable state and a larger set of equational theories than other approaches as detailed below.

Various other tools exist for verifying notions of observational equivalence. APTE $[9,11]$ and AKISS [8] both verify trace equivalence, but are limited to a bounded number of sessions. Moreover, AKISS does not support nontrivial else branches or private channels. ProVERIF [7] verifies observational equivalence in the applied $\pi$-calculus for an unbounded number of sessions, but it cannot handle mutable state [3], for example, a protocol that switches between the states $a$ and $b$. Extensions for ProVERIF that can deal with Diffie-Hellman equational theories [23] do not support observational equivalence. Note that our approach's restriction to bi-systems is similar to PROVERIF's restriction to bi-processes. SPEC [29] verifies open bisimulation in the spi-calculus, but unlike our approach it only supports a fixed number of cryptographic primitives and is limited to a bounded number of sessions.

In contrast to the above, there are tools like STATVERIF [3] and SAPIC [22] that support mutable state. However, they cannot verify observational equivalence. Similarly, TAMARIN, which is used as SAPIC's back-end, supports mutable state, an unbounded number of sessions, and also Diffie-Hellmann equational theories. However, prior to our extension, it 
could not prove any notion of observational equivalence.

Another multiset rewriting-based approach that supports observational equivalence is Maude-NPA [27]. It creates the synchronous product of two very similar protocols, similar to our use of bi-systems. Their approach suffers from termination problems [27] and thus presents only attacks.

As future work, we plan to extend our approach so that the verification of observational equivalence is also possible when one rule must be matched by a different rule, or even by multiple rules. We will also tackle protocols with loops, where proofs will likely require induction. Moreover, we intend to look at larger protocols, such as authenticated key exchange protocols with perfect forward secrecy, such as NAXOS and its variants.

\section{REFERENCES}

[1] Tamarin - tool and extended papers. http://www . infsec.ethz.ch/research/software/tamarin.html.

[2] Martín Abadi and Cédric Fournet. Mobile values, new names, and secure communication. In Proceedings of the 28th Symposium on Principles of Programming Languages (POPL'01), pages 104-115, New York, 2001. ACM.

[3] Myrto Arapinis, Joshua Phillips, Eike Ritter, and Mark Dermot Ryan. Statverif: Verification of stateful processes. Journal of Computer Security, 22(5):743-821, 2014.

[4] David Basin, Cas Cremers, and Catherine Meadows. Model checking security protocols. In Handbook of Model Checking, chapter 24. Springer, 2015. To appear.

[5] Mihir Bellare, Anand Desai, David Pointcheval, and Phillip Rogaway. Relations among notions of security for public-key encryption schemes. In CRYPTO, volume 1462 of LNCS, pages 26-45. Springer, 1998.

[6] Mihir Bellare and Phillip Rogaway. Entity authentication and key distribution. In CRYPTO, volume 773 of LNCS, pages 232-249. Springer, 1993.

[7] Bruno Blanchet, Martín Abadi, and Cédric Fournet. Automated verification of selected equivalences for security protocols. Journal of Logic and Algebraic Programming, 75(1):3-51, February-March 2008.

[8] Rohit Chadha, Ştefan Ciobâcă, and Steve Kremer. Automated verification of equivalence properties of cryptographic protocols. In Helmut Seidl, editor, ESOP, volume 7211 of $L N C S$, pages 108-127. Springer, 2012.

[9] Vincent Cheval. APTE: An algorithm for proving trace equivalence. In TACAS, volume 8413 of $L N C S$, pages 587-592. Springer, 2014.

[10] Vincent Cheval and Bruno Blanchet. Proving more observational equivalences with ProVerif. In Principles of Security and Trust (POST), volume 7796 of LNCS, pages 226-246. Springer, 2013.

[11] Vincent Cheval, Véronique Cortier, and Stéphanie Delaune. Deciding equivalence-based properties using constraint solving. Theor. Comput. Sci., 492:1-39, 2013.

[12] Y. Chevalier, R. Küsters, M. Rusinowitch, and M. Turuani. Deciding the security of protocols with Diffie-Hellman exponentiation and products in exponents. In FSTTCS 2003, volume 2914 of $L N C S$, pages 124-135. Springer, 2003.

[13] Hubert Comon-Lundh and Stéphanie Delaune. The finite variant property: How to get rid of some algebraic properties. In Jürgen Giesl, editor, RTA, volume 3467 of LNCS, pages 294-307. Springer, 2005.

[14] Stéphanie Delaune, Steve Kremer, and Mark Ryan. Verifying privacy-type properties of electronic voting protocols. Journal of Computer Security, 17:435-487, December 2009.

[15] Stéphanie Delaune, Steve Kremer, Mark Dermot Ryan, and Graham Steel. Formal analysis of protocols based on TPM state registers. In $C S F$, pages $66-80$. IEEE, 2011.

[16] Jannik Dreier, Pascal Lafourcade, and Yassine Lakhnech. Defining privacy for weighted votes, single and multi-voter coercion. In ESORICS, volume 7459 of LNCS, pages 451-468. Springer, 2012.

[17] Jannik Dreier, Pascal Lafourcade, and Yassine Lakhnech. A formal taxonomy of privacy in voting protocols. In Proceedings of IEEE International Conference on Communications (ICC'12), pages 6710-6715, Ottawa, ON, Canada, 2012. IEEE.

[18] Jannik Dreier, Pascal Lafourcade, and Yassine Lakhnech. Formal verification of e-auction protocols. In Proceedings of the 2nd Conference on Principles of Security and Trust (POST'13), volume 7796 of LNCS, pages 247-266, Rome, Italy, 2013. Springer Verlag.

[19] Santiago Escobar, Ralf Sasse, and José Meseguer. Folding variant narrowing and optimal variant termination. Journal of Logic and Algebraic Programming, 81(7-8):898-928, 2012.

[20] Martin Feldhofer, Sandra Dominikus, and Johannes Wolkerstorfer. Strong authentication for RFID systems using the aes algorithm. In Cryptographic Hardware and Embedded Systems-CHES 2004, pages 357-370. Springer, 2004.

[21] Michèle Feltz and Cas Cremers. On the limits of authenticated key exchange security with an application to bad randomness. Cryptology ePrint Archive, Report 2014/369, 2014.

[22] Steve Kremer and Robert Künnemann. Automated analysis of security protocols with global state. In 2014 IEEE Symposium on Security and Privacy, SP 2014, Berkeley, CA, USA, May 18-21, 2014, pages 163-178. IEEE Computer Society, 2014.

[23] Ralf Küsters and Tomasz Truderung. Using ProVerif to analyze protocols with Diffie-Hellman exponentiation. In Computer Security Foundations Symposium (CSF), pages 157-171. IEEE, 2009.

[24] Brian LaMacchia, Kristin Lauter, and Anton Mityagin. Stronger security of authenticated key exchange. In Provable Security, pages 1-16. Springer, 2007.

[25] Laurie Law, Alfred Menezes, Minghua Qu, Jerry Solinas, and Scott Vanstone. An efficient protocol for authenticated key agreement. Designs, Codes and Cryptography, 28:119-134, 2003.

[26] Simon Meier, Benedikt Schmidt, Cas Cremers, and David Basin. The TAMARIN Prover for the Symbolic Analysis of Security Protocols. In $C A V$, volume 8044 of $L N C S$, pages 696-701. Springer, 2013.

[27] Sonia Santiago, Santiago Escobar, Catherine 
Meadows, and José Meseguer. A formal definition of protocol indistinguishability and its verification using Maude-NPA. In Security and Trust Management (STM) 2014, pages 162-177. Springer, 2014.

[28] Benedikt Schmidt, Simon Meier, Cas Cremers, and David Basin. Automated analysis of Diffie-Hellman protocols and advanced security properties. In Computer Security Foundations Symposium (CSF), pages 78-94. IEEE, 2012.

[29] Alwen Tiu and Jeremy E. Dawson. Automating open bisimulation checking for the spi calculus. In $C S F$, pages 307-321. IEEE Computer Society, 2010.

[30] Ton Van Deursen, Sjouke Mauw, and Saša Radomirović. Untraceability of RFID protocols. In Information Security Theory and Practices. Smart Devices, Convergence and Next Generation Networks, pages 1-15. Springer, 2008. 


\section{APPENDIX}

\section{A. TAMARIN}

TAMARIN is based on a type system where all messages are of the (top) sort $m s g$, which includes as subsorts public values of type $p u b$ and fresh values of type $f r$. Cryptographic primitives and algebraic properties of terms are modeled by equations, for example $\operatorname{dec}(\operatorname{enc}(m, k), k)=m$ models that given a symmetric encryption of a message $m$, its decryption using the correct key $k$ gives $m$.

In TAMARIN, a protocol is modeled using multiset rewrite rules, and a set of facts that captures the state of the protocol, the adversarial knowledge, and the state of the network. In particular, the set of facts includes the special facts $\mathrm{K}(t)$, $\operatorname{Fr}(t), \operatorname{Frl}(t)$, Out $(t)$, and $\ln (t)$. The persistent fact $\mathrm{K}(t)$ represents the intruder's knowledge, i.e., the intruder knows the term $t$. Note that, in the implementation, $\mathrm{K}(t)$ is split into different facts to facilitate automated reasoning, e.g., $\mathrm{K}_{e}^{\uparrow}(t)$ and $\mathbf{K}_{e}^{\downarrow}(t)$, which we describe in more detail later. The linear facts $\operatorname{Fr}(x)$ (and $\operatorname{Frl}(x)$ ) express that $x$ is a freshly generated term, usually called a fresh variable, by the protocol (respectively the intruder), and the linear facts Out $(t)$ and $\ln (t)$ model the output or input of a term $t$ by the protocol, corresponding to Out $\operatorname{Sys}_{S y}(t)$ and $\ln _{S y s}(t)$ in the initial model.

In TAMARIN we consider three types of rules: fresh rules used to generate fresh values, message deduction rules, and the protocol and intruder rules. The fresh rules FRESH $_{\text {Sys }}=$ $\{[]-[\} \operatorname{Fr}(x: f r)\}$ and $\operatorname{FrESH}_{E n v}=\{([]-[\} \rightarrow \operatorname{Frl}(x:$ fr $)\}$ are the only rules that can be used to generate fresh values. The first rule is used by the protocol and the second one by the intruder. To ensure that the generated values are fresh, we require that each instance is unique, i.e., each fresh value $x$ can be generated only once during the execution of the protocol, either by the protocol or by the intruder. We combine both fresh rules into FRESH $=\mathrm{FRESH}_{S y s} \cup \mathrm{FrESH}_{E n v}$. There are also a number of distinguished facts that are used only in actions to label transitions.

The message deduction rules characterizing the intruder are given in Figure 7 . The two communication rules represent the interface rules $I F$ as defined in Section 3; we simply renamed the facts: Out instead of Out ${ }_{S y s}, \ln$ instead of $\ln _{S y s}$, $\mathrm{K}^{\uparrow}$ instead of Out ${ }_{E n v}$, and $\mathrm{K}^{\downarrow}$ instead of $\ln _{E n v}$. We do this renaming because the $\mathrm{K}^{\uparrow}$ and $\mathrm{K}^{\downarrow}$ notation is needed to restrict the applicable rules, as seen in the rest of the figure. The coerce rule allows the intruder to convert a $\mathrm{K}^{\downarrow}$-fact into a $\mathrm{K}^{\uparrow}$-fact, but not vice versa. The idea is that the intruder will first decompose a message he receives (as a $\mathrm{K}^{\downarrow}$-fact) using the deconstruction rules, apply the coerce-rule, and then construct new messages using the construction rules working on $\mathrm{K}^{\uparrow}$-facts. However, once he has applied the coerce-rule, he can no longer apply deconstruction rules. This prevents loops where the intruder constructs messages that he later deconstructs, only to construct the same message again, and so on.

The construction rules enable the intruder to apply functions to terms he knows, in particular exponentiation, encryption, decryption, hashing, tuple construction, multiplication, and potentially other user-defined functions (not shown in the figure, but supported in Tamarin). Moreover, these rules allow the intruder to use public values, constants, and fresh values. The deconstruction rules allow the intruder to decompose terms he receives, for example tuples, apply the multiplicative inverse, decrypt ciphertexts for which he possesses the key, or apply other user-defined subtermconvergent theories (again not shown in the figure). Finally the exponentiation rules formalize Diffie-Hellman exponentiation modulo AC. This particular representation, although verbose, allows for automatic reasoning (for more details see [28]).

The variants of a term $t$ provide a concise representation of all possible instantiations of $t$. The use of $D H, A C$-variants of the exponentiation rule thus allows us to restrict exponentiation to the 42 variants, giving rise to 42 rules as mentioned. More detail on variants are provided below.

The equality rule is added to ensure that the intruder cannot distinguish terms output by the protocol, similar to e.g., static equivalence in the applied $\pi$-calculus [7]. The rule has no conclusion facts since we are only concerned about its applicability, corresponding to an equality that holds. By the definition of observational equivalence, each intruder rule must be matched by itself. This ensures that if an equality holds on one side (i.e., the rule is applicable), it also holds on the other side. Note that we match a $\mathrm{K}^{\downarrow}$-fact with a $\mathrm{K}^{\uparrow}$-fact. The $\mathrm{K}^{\uparrow}$-fact allows the intruder to construct more complex terms by, e.g., applying functions, while the $\mathrm{K}^{\downarrow}$-fact limits the search space to prevent the intruder from building unnecessarily complex terms. For example, if two terms are equal, their hash values are equal, but additionally testing the hashes does not give the intruder additional decision power.

A protocol rule is a multiset rewriting rule $i d: l-[a \mapsto r$ such that (P1) it contains no fresh names, (P2) K, Out, and $\mathrm{Frl}$ facts do not occur in $l$, (P3) $\mathrm{K}$, In, Fr and Frl facts do not occur in $r$, and $(\mathrm{P} 4) \operatorname{vars}(r) \subseteq \operatorname{vars}(l) \cup \mathcal{V}_{p u b}$. A protocol is specified by a finite set of protocol rules. Note that these rules encompass both the rules executed by the honest participants and adversary capabilities, like revealing long-term keys. Condition (P1) and the restriction on the usage of Fr and Frl facts from (P3), which also hold for the message deduction rules, ensure that all fresh names originate from instances of the FRESH rules.

\section{A.1 Observational Equivalence}

To prove observational equivalence in TAMARIN, we use the algorithm provided in Figure 4 in Section 4, which we now further explain. For efficiency reasons, TAMARIN merges both fresh rules and facts, but includes a further restriction on dependency graphs:

DG4: The FRESH instances are unique.

As the following theorem shows, merging the different fresh facts is sound. The proof is similar to the proof of Theorem 1.

THEOREM 2. Let $S$ be a protocol bi-system. Then

$$
\begin{gathered}
L(S) \sim{ }_{D G, I F \cup{ }_{\text {RRESH }} \cup N D} R(S) \\
\Downarrow \\
\left(L(S) \cup \mathrm{FRESH}_{S y s}\right) \sim_{\mathrm{FRESH}_{E n v} \cup N D}\left(R(S) \cup \mathrm{FRESH}_{S y s}\right) .
\end{gathered}
$$

Proof. Consider the multiset rewrite systems $L=L(S) \cup$ $I F \cup \mathrm{FRESH} \cup N D$ and $R=R(S) \cup I F \cup \mathrm{FRESH} \cup N D$, and the following relation $\mathcal{R}$ :

$$
\begin{array}{r}
\mathcal{R}=\left\{\left(\mathcal{S}_{A}, \mathcal{S}_{B}\right) \mid \mathcal{S}_{A}=\operatorname{state}\left(d g_{L}\right), \mathcal{S}_{B}=\operatorname{state}\left(d g_{R}\right),\right. \\
\left.d g_{R} \in \text { mirrors }\left(d g_{L}\right), d g_{L} \in \operatorname{dgraphs}(L)\right\} \\
\cup \quad\left\{\left(\mathcal{S}_{A}, \mathcal{S}_{B}\right) \mid \mathcal{S}_{A}=\operatorname{state}\left(d g_{L}\right), \mathcal{S}_{B}=\operatorname{state}\left(d g_{R}\right),\right. \\
\left.d g_{L} \in \text { mirrors }\left(d g_{R}\right), d g_{R} \in \operatorname{dgraphs}(R)\right\}
\end{array}
$$


Coerce rule:

$$
\text { CoErce } \frac{\mathrm{K}_{e}^{\downarrow}(x)}{\mathrm{K}_{e}^{\uparrow}(x)}
$$

Communication rules:

$$
\operatorname{IRECV} \frac{\operatorname{Out}(x)}{\mathrm{K}_{\exp }^{\downarrow}(x)} \quad \operatorname{ISEND} \frac{\mathrm{K}_{e}^{\uparrow}(x)}{\ln (x)}[\mathrm{K}(x)]
$$

Equality rule:

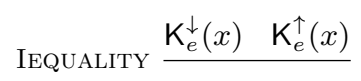

Construction rules:

$$
\begin{aligned}
& \operatorname{EXP} \uparrow \frac{\mathrm{K}_{\exp }^{\uparrow}(x) \mathrm{K}_{e}^{\uparrow}(y)}{\mathrm{K}_{\text {noexp }}^{\uparrow}\left(x^{\wedge} y\right)} \quad \operatorname{PUB} \frac{\operatorname{Crl}(x: f r)}{\mathrm{K}_{\exp }^{\uparrow}(x: p u b)} \quad \operatorname{CFRESH} \frac{\operatorname{INV} \uparrow \frac{\mathrm{K}_{e}^{\uparrow}(x)}{\mathrm{K}_{\exp }^{\uparrow}(x: f r)} \quad \text { ONE } \frac{\mathrm{K}_{e_{1}}^{\uparrow}(x)}{\mathrm{K}_{\exp }^{\uparrow}(1)} \quad \mathrm{K}_{e_{2}}^{\uparrow}(y)}{\mathrm{K}_{\exp }^{\uparrow}(\operatorname{enc}(x, y))} \quad \frac{\mathrm{K}_{e_{1}}^{\uparrow}(x)}{\mathrm{K}_{\exp }^{\uparrow}(\operatorname{dec}(x, y))} \\
& \frac{\mathrm{K}_{e}^{\uparrow}(x)}{\mathrm{K}_{\exp }^{\uparrow}(\mathrm{h}(x))} \quad \frac{\mathrm{K}_{e}^{\uparrow}(x)}{\mathrm{K}_{\exp }^{\uparrow}(\mathrm{fst}(x))} \quad \frac{\mathrm{K}_{e}^{\uparrow}(x)}{\mathrm{K}_{\exp }^{\uparrow}(\operatorname{snd}(x))} \quad \operatorname{PAIR} \uparrow \frac{\mathrm{K}_{e_{1}}^{\uparrow}(x) \mathrm{K}_{e_{2}}^{\uparrow}(y)}{\mathrm{K}_{\exp }^{\uparrow}(\langle x, y\rangle)} \quad \operatorname{MULT} \uparrow \frac{\mathrm{K}_{e_{1}}^{\uparrow}\left(x_{1}\right) \ldots \mathrm{K}_{e_{n}}^{\uparrow}\left(x_{n}\right) \quad \mathrm{K}_{e_{n+1}}^{\uparrow}\left(x_{n+1}\right) \ldots \mathrm{K}_{e_{l}}^{\uparrow}\left(x_{l}\right)}{\mathrm{K}_{\exp }^{\uparrow}\left(\left(x_{1} * \ldots * x_{n}\right) *\left(x_{n+1} * \ldots * x_{l}\right)^{-1}\right)}
\end{aligned}
$$

Deconstruction rules:

$$
\operatorname{EXP} \downarrow \frac{\mathrm{K}_{\exp }^{\downarrow}\left(x^{\wedge} y\right) \mathrm{K}_{e}^{\uparrow}\left(y^{-1}\right)}{\mathrm{K}_{\text {noexp }}^{\downarrow}(x)} \quad \operatorname{EXP} \downarrow \frac{\mathrm{K}_{\exp }^{\downarrow}\left(x^{\wedge} y^{-1}\right) \mathrm{K}_{e}^{\uparrow}(y)}{\mathrm{K}_{\text {noexp }}^{\downarrow}(x)} \quad \operatorname{EXP} \downarrow \frac{\mathrm{K}_{\exp }^{\downarrow}\left(x^{\wedge}\left(y * z^{-1}\right)\right) \mathrm{K}_{e}^{\uparrow}\left(y^{-1} * z\right)}{\mathrm{K}_{\text {noexp }}^{\downarrow}(x)}
$$

$$
\operatorname{FST} \downarrow \frac{\mathrm{K}_{e}^{\downarrow}(\langle x, y\rangle)}{\mathrm{K}_{\exp }^{\downarrow}(x)} \quad \operatorname{SND} \downarrow \frac{\mathrm{K}_{e}^{\downarrow}(\langle x, y\rangle)}{\mathrm{K}_{\exp }^{\downarrow}(y)} \quad \operatorname{INV} \downarrow \frac{\mathrm{K}_{e}^{\downarrow}\left(x^{-1}\right)}{\mathrm{K}_{\exp }^{\downarrow}(x)} \quad \frac{\mathrm{K}_{e_{1}}^{\downarrow}(\operatorname{enc}(x, y)) \quad \mathrm{K}_{e_{2}}^{\uparrow}(y)}{\mathrm{K}_{\exp }^{\downarrow}(x)}
$$

$$
\text { Exponentiation rules: } \quad \operatorname{Exp} \downarrow \frac{\mathrm{K}_{\exp }^{\downarrow}\left(x^{\wedge} y\right) \mathrm{K}_{e}^{\uparrow}(z)}{\mathrm{K}_{\text {noexp }}^{\downarrow}\left(x^{\wedge}(y * z)\right)} \quad \operatorname{ExP} \downarrow \frac{\mathrm{K}_{\exp }^{\downarrow}\left(x^{\wedge} y\right) \mathrm{K}_{e}^{\uparrow}\left(y^{-1} * z\right)}{\mathrm{K}_{\text {noexp }}^{\downarrow}\left(x^{\wedge} z\right)} \quad \cdots \quad \operatorname{Exp} \downarrow \frac{\mathrm{K}_{\exp }^{\downarrow}\left(x^{\wedge}\left(y * z^{-1}\right)\right) \mathrm{K}_{e}^{\uparrow}\left(a * b^{-1}\right)}{\mathrm{K}_{\text {noexp }}^{\downarrow}\left(x^{\wedge}\left(y * a *(z * b)^{-1}\right)\right)}
$$

Figure 7: Normal message deduction rules $N D$. Rules containing variables $e$ or $e_{i}$ denote all variants where these are replaced by noexp or exp. Rules containing $n$ and $l$ denote all variants for $n \geq 1$ and $l \geq 2$. There are 42 exponentiation rules computed from the $D H, A C$-variants of the exponentiation rule.
(1) $\operatorname{dec}(\operatorname{enc}(m, k), k) \simeq m$
(6) $x * 1 \simeq x$
(2) $\operatorname{fst}(\langle x, y\rangle) \simeq x$
(7) $x * x^{-1} \simeq 1$
(3) $\operatorname{snd}(\langle x, y\rangle) \simeq y$
(8) $\left(x^{-1}\right)^{-1} \simeq x$
(4) $x *(y * z) \simeq(x * y) * z$
(9) $\left(x^{\wedge} y\right)^{\wedge} z \simeq x^{\wedge}(y * z)$
(5) $x * y \simeq y * x$
(10) $x^{\wedge} 1 \simeq x$

Figure 8: Equations that constitute $E_{D H}$.
(1) $\left(x^{-1} * y\right)^{-1} \simeq x * y^{-1}$
(6) $1^{-1} \simeq 1$
(2) $x^{-1} * y^{-1} \simeq(x * y)^{-1}$
(7) $x * 1 \simeq x$
(3) $x *(x * y)^{-1} \simeq y^{-1}$
(8) $\left(x^{-1}\right)^{-1} \simeq x$
(4) $x^{-1} *\left(y^{-1} * z\right) \simeq(x * y)^{-1} * z$
(9) $x *\left(x^{-1} * y\right) \simeq y$
(5) $(x * y)^{-1} *(y * z) \simeq x^{-1} * z$
(10) $x * x^{-1} \simeq 1$

Figure 9: Lankford's presentation of the abelian group axioms

First note that $(\emptyset, \emptyset) \in \mathcal{R}$. Now we show that $\mathcal{R}$ is an observational equivalence relation as defined in Def. 1. For this, we must show the following for all states $\left(\mathcal{S}_{A}, \mathcal{S}_{B}\right) \in \mathcal{R}$ :

1. If $\mathcal{S}_{A} \underset{r}{\stackrel{l}{\rightarrow}} \mathcal{S}_{A}^{\prime}$ and $r$ is the recipe of a rule in $I F \cup \mathrm{FRESH} \cup$ $N D$, then there exists $l^{\prime}$ and $\mathcal{S}_{B}^{\prime}$ such that $\mathcal{S}_{B} \underset{r}{\stackrel{l^{\prime}}{\rightarrow}} \mathcal{S}_{B}^{\prime}$, and $\left(\mathcal{S}_{A}^{\prime}, \mathcal{S}_{B}^{\prime}\right) \in \mathcal{R}$.

2. If $\mathcal{S}_{A} \underset{r}{\stackrel{l}{\rightarrow}} \mathcal{S}_{A}^{\prime}$ and $r$ is the recipe of a rule in $L(S)$, then there exist a recipe $r^{\prime}$ of a rule in $R(S), l^{\prime}$, and $\mathcal{S}_{B}^{\prime}$ such that $\mathcal{S}_{B} \underset{r^{\prime}}{\stackrel{l^{\prime}}{\longrightarrow}} \mathcal{S}_{B}^{\prime}$, and $\left(\mathcal{S}_{A}^{\prime}, \mathcal{S}_{B}^{\prime}\right) \in \mathcal{R}$. and the analogous statements for the symmetric cases.

Let $L^{\prime}=L(S) \cup I F \cup \mathrm{FRESH}_{S y s} \cup N D^{\prime}$ and $R^{\prime}=R(S) \cup$ $I F \cup \mathrm{FRESH}_{S y s} \cup N D^{\prime}$. Given a dependency graph $d g$, let mergeFresh $(d g)$ denote the dependency graph obtained by replacing all Frl facts with Fr facts and all instances of the FRESH $_{E n v}$-rule with the same instance (i.e., creating the same fresh name) of the $\mathrm{FRESH}_{E n v}$-rule.

1. Assume $\left(\mathcal{S}_{A}, \mathcal{S}_{B}\right) \in \mathcal{R}, \mathcal{S}_{A} \underset{r}{\stackrel{l}{\rightarrow}} \mathcal{S}_{A}^{\prime}$ for a rule instance $r i$, and $r$ is the recipe of a rule in $I F \cup F R E S H \cup N D$. Then, by the definition of $\mathcal{R}$, there is a dependency graph $d g_{L} \in$ $\operatorname{dgraphs}(L)$ with $\mathcal{S}_{A}=\operatorname{state}\left(d g_{L}\right)$, and a dependency graph $d g_{R} \in \operatorname{dgraphs}(R)$ with $\mathcal{S}_{B}=\operatorname{state}\left(d g_{R}\right)$.

- Suppose that $r i$ is an instance of the $\operatorname{FRESH}_{E n v}$ rule. Then $\mathcal{S}_{B} \underset{r}{\stackrel{l^{\prime}}{\rightarrow}} \mathcal{S}_{B}^{\prime}$ for the same instance $r i$ of the FRESHEnv rule, as the rule has no premises. In particular, the variable is instantiated with the same fresh name as in $r i$, hence we have the same recipe. Moreover, $\left(\mathcal{S}_{A}^{\prime}, \mathcal{S}_{B}^{\prime}\right) \in \mathcal{R}$ since there are mirroring dependency graphs for $\mathcal{S}_{A}^{\prime}$ and $\mathcal{S}_{B}^{\prime}$; we can simply extend $d g_{L}$ and $d g_{R}$ with $r i$.

- Suppose that $r i$ was an instance of the construction rule CFresh in $N D$. This means that $\mathcal{S}_{A}$ contains a Frl fact, which can only have been created using the FRESH Env $_{\text {rule. As }} \mathcal{S}_{A}$ and $\mathcal{S}_{B}$ have mirroring dependency graphs, $\mathcal{S}_{B}$ contains the same Frl fact, since it must contain the same FRESH Env $_{\text {r }}$ rule instance. Hence $\mathcal{S}_{B} \underset{r}{\stackrel{l}{\rightarrow}} \mathcal{S}_{B}^{\prime}$ for the same instance $r i$ of the FRESH rule. Moreover, $\left(\mathcal{S}_{A}^{\prime}, \mathcal{S}_{B}^{\prime}\right) \in \mathcal{R}$ since there are mirroring dependency graphs for $\mathcal{S}_{A}^{\prime}$ and $\mathcal{S}_{B}^{\prime}$ : we can extend $d g_{L}$ with $r i$ and the correct edge, and use the same extension (including the edge) to extend $d g_{R}$ to have two mirroring depen- 
dency graphs with the right states.

- Suppose that $r i$ is an instance of another rule in $I F \cup$ FRESH $\cup N D$. Since in $\mathcal{S}_{A}$ the transition $\mathcal{S}_{A} \underset{r}{\stackrel{l}{\rightarrow}}$ $\mathcal{S}_{A}^{\prime}$ is possible, $d g_{L}$ can be extended to $d g_{L}^{\prime}$ with the rule instance $r i$ corresponding to this transition, and state $\left(d g_{L}^{\prime}\right)=\mathcal{S}_{A}^{\prime}$. Then mergeFresh $\left(d g_{L}^{\prime}\right) \in$ $\operatorname{dgraphs}\left(L^{\prime}\right)$, and by $L(S) \sim_{D G} R(S)$ for all possible instantiations of new diff variables, the corresponding dependency graph $d g_{R}^{\prime} \in \operatorname{dgraphs}\left(R^{\prime}\right)$. By the definition of $\mathcal{R}$, the instantiations of the new variables (including the new diff-variables) in $d g_{R}$ correspond to the instantiations of one $d g_{R}^{\prime} \in$ mirrors $\left(d g_{L}^{\prime}\right)$. Then, by construction of mirrors $\left(d g_{L}^{\prime}\right)$, $d g_{R}^{\prime}$ is identical to mergeFresh $\left(d g_{R}\right)$ except for the last rule instance $r i^{\prime}$. Moreover, by construction of mirrors $\left(d g_{L}^{\prime}\right), r i^{\prime}$ is an instance of the rule with the same identifier. Since the dependency graph $d g_{R}^{\prime}$ has the same structure $D$ as $d g_{L}^{\prime}$ and all rules in $I F \cup \mathrm{FRESH}_{S y s} \cup N D$ have no new diff-variables, there exists a transition $\mathcal{S}_{B} \underset{r}{\stackrel{l^{\prime}}{\rightarrow}} \mathcal{S}_{B}^{\prime}$ with the same recipe as ri. Moreover, $\left(\mathcal{S}_{A}^{\prime}, \mathcal{S}_{B}^{\prime}\right) \in \mathcal{R}$ since there are mirroring dependency graphs for $\mathcal{S}_{A}^{\prime}$ and $\mathcal{S}_{B}^{\prime}$.

The symmetric case is analogous.

2. Now assume $\left(\mathcal{S}_{A}, \mathcal{S}_{B}\right) \in \mathcal{R}, \mathcal{S}_{A} \underset{r}{\stackrel{l}{\rightarrow}} \mathcal{S}_{A}^{\prime}$ and $r$ is the recipe of a rule in $L(S)$. Then, by definition of $\mathcal{R}$, there is a dependency graph $d g_{L} \in \operatorname{dgraphs}(L)$ with $\mathcal{S}_{A}=\operatorname{state}\left(d g_{L}\right)$. Since in this state the transition $\mathcal{S}_{A} \underset{r}{\stackrel{l}{\rightarrow}} \mathcal{S}_{A}^{\prime}$ is possible, $d g_{L}$ can be extended to $d g_{L}^{\prime}$ with the rule instance $r i$ corresponding to this transition, and state $\left(d g_{L}^{\prime}\right)=\mathcal{S}_{A}^{\prime}$. Then mergeFresh $\left(d g_{L}^{\prime}\right) \in$ $\operatorname{dgraphs}\left(L^{\prime}\right)$, and by $L(S) \sim_{D G} R(S)$ we have that for all possible instantiations of new diff variables, the corresponding dependency graph $d g_{R}^{\prime} \in \operatorname{dgraphs}\left(R^{\prime}\right)$. By definition of $\mathcal{R}$, there is a dependency graph $d g_{R} \in$ $\operatorname{dgraphs}(R)$ with $\mathcal{S}_{B}=\operatorname{state}\left(d g_{R}\right)$, where the instantiations of the new variables (including the new diffvariables) correspond to the instantiations of one $d g_{R}^{\prime} \in$ mirrors $\left(d g_{L}^{\prime}\right)$. Then, by construction of mirrors $\left(d g_{L}^{\prime}\right)$, this graph $d g_{R}^{\prime}$ is identical to mergeFresh $\left(d g_{R}\right)$ except for the last rule instance. By assumption, $r i$ was an instance of a rule in $L(S)$. Then, by construction of $\operatorname{mirrors}\left(d g_{L}^{\prime}\right)$, the last rule instance $r i^{\prime}$ in $d g_{R}^{\prime}$ is an instance of the rule with the same identifier. Hence there exists a transition $\mathcal{S}_{B} \underset{r^{\prime}}{\stackrel{l^{\prime}}{\longrightarrow}} \mathcal{S}_{B}^{\prime}$. Moreover, $\left(\mathcal{S}_{A}^{\prime}, \mathcal{S}_{B}^{\prime}\right) \in \mathcal{R}$ since there are mirroring dependency graphs for $\mathcal{S}_{A}^{\prime}$ and $\mathcal{S}_{B}^{\prime}$

Again, the symmetric case is analogous.

\section{A.2 Restricted Normal Dependency Graphs}

To facilitate automated reasoning, our algorithm only considers restricted normal dependency graphs, a normalized variant of dependency graphs, where terms are in normal form and certain redundant intruder steps are eliminated.

We call the equational theory generated by Equations (45) from Figure $8 A C$ and the rewriting system obtained by orienting Equations (1-3,9-10) from Figure 8 and all equations from Figure 9 from left to right $D H . D H \uplus A C$ is an equational presentation of $E_{D H}$ and $D H$ is $A C$-convergent and $A C$-coherent. We can therefore define $t \downarrow_{D H}$ as the normal form of $t$ with respect to $D H, A C$-rewriting and have $t=E_{D H} s$ iff $t \downarrow_{D H}=A_{A C} s \downarrow_{D H}$. We say that $t$ is $\downarrow_{D H}$-normal if $t={ }_{A C} t \downarrow_{D H}$. Moreover we call a set of facts $F \downarrow_{D H}$-normal if all terms inside the facts are $\downarrow_{D H}$-normal, and $F \downarrow_{D H}$ denotes $F$ with all terms normalized.

Note that $E_{D H}$ has the finite variant property [13] for this presentation, which allows us to perform symbolic reasoning about normalization. More precisely, for all terms $t$, there is a finite set of substitutions $\left\{\tau_{1}, \ldots, \tau_{k}\right\}$ such that for all substitutions $\sigma$, there is an $i \in\{1, \ldots, k\}$ and a substitution $\sigma^{\prime}$ with $(t \sigma) \downarrow_{D H}={ }_{A C}\left(\left(t \tau_{i}\right) \downarrow_{D H}\right) \sigma^{\prime}$ and $(x \sigma) \downarrow_{D H}={ }_{A C} x \tau_{i} \sigma^{\prime}$ for all $x \in \operatorname{vars}(t)$. We call $\left\{\left(t \tau_{i \downarrow_{D H}}, \tau_{i}\right) \mid 1 \leq i \leq k\right\}$ a complete set of $D H, A C$-variants of $t$. For a given term $t$, we use folding variant narrowing [19] to compute such a set, which we denote by $\lceil t\rceil^{D H}$. Overloading notation, we also denote $\left\{s \mid(s, \tau) \in\lceil t\rceil^{D H}\right\}$ by $\lceil t\rceil^{D H}$. It is straightforward to extend these notions to multiset rewriting rules by considering rules as terms and the required new function symbols as free.

We define the input components of a term $t$ as inp $(t)$, such that $\operatorname{inp}\left(t^{-1}\right)=\operatorname{inp}(t), \operatorname{inp}\left(\left\langle t_{1}, t_{2}\right\rangle\right)=\operatorname{inp}\left(t_{1}\right) \cup \operatorname{inp}\left(t_{2}\right)$, $\operatorname{inp}\left(t_{1} * t_{2}\right)=\operatorname{inp}\left(t_{1}\right) \cup i n p\left(t_{2}\right)$, and $\operatorname{inp}(t)=\{t\}$ otherwise. Intuitively, inp $(t)$ consists of the maximal subterms of $t$ that are not products, pairs, or inverses.

Definition 5 (Restricted Normal DG). A restricted normal dependency graph for a protocol $R$ is a dependency graph dg such that

$$
d g \in \operatorname{dgraphs}_{A C}\left(\lceil R\rceil^{D H} \cup I F \cup \mathrm{FRESH}_{S y s} \cup N D^{\prime}\right)
$$

and the following conditions are satisfied (in addition to DG1-DG4):

N1 All rule instances in I are $\downarrow_{D H}$-normal.

N2 No instance of COERCE deduces a pair or an inverse.

N3 There is no multiplication rule that has a premise fact of the form $K_{e}^{\uparrow}(t * s)$.

$N 5$ ' If there are two conclusions $c$ and $c^{\prime}$ with conclusion facts $K_{e}^{\uparrow}(m)$ and $K_{e^{\prime}}^{\uparrow}\left(m^{\prime}\right)$ such that $m={ }_{A C} m^{\prime}$, then $c=c^{\prime}$.

$N 5$ " If there is a conclusion $(i, 1)$ with fact $K_{e}^{\uparrow}(m)$, a conclusion $(j, 1)$ with fact $K_{e^{\prime}}^{\downarrow}\left(m^{\prime}\right)$ such that $m={ }_{A C} m^{\prime}$ and $i<j$, then for all $k$ such that $(j, 1) \longmapsto(k, 1) \in D, k$ is an instance of the IEquality rule.

$N 7$ For all nodes $K_{\text {exp }}^{\downarrow}\left(s_{1}\right), K_{e}^{\uparrow}\left(t_{1}\right)-[\} \rightarrow K_{\text {noexp }}^{\downarrow}\left(s_{2}{ }^{\wedge} t_{2}\right)$ such that $s_{2}$ is of sort pub, inp $\left(t_{2}\right) \nsubseteq \operatorname{inp}\left(t_{1}\right)$.

We denote the set of all normal dependency graphs of $P$ by $n d g r a p h s(P)$.

Note that, in contrast to normal dependency graphs in previous versions of TAMARIN [28], our restricted normal dependency graphs have fewer and also different restrictions to ensure soundness. Notably we have to remove restrictions N4 and N6, and weakened N5. Intuitively, N4, N5, and N6 ensured that the intruder could not deduce the same term (modulo the equational theory) multiple times. However, in the context of observational equivalence, and notably the equality rule, it is crucial that the adversary is able to deduce a (potentially) identical term twice (maybe from different sources), for example to test whether the protocol outputs 
the same value twice. Thus we had to remove or weaken these restrictions. In particular we weakened N5 to N5' and N5" to allow the adversary to deduce the same term $m$ in a $\mathrm{K}_{e}^{\downarrow}(m)$ fact multiple times. However, N5" ensures that these deductions are only used for the equality rule, as coercing the same term to obtain $\mathrm{K}_{e}^{\uparrow}(m)$ multiple times is unnecessary.

Analogous to dependency graph equivalence, we can now define Restricted Dependency Graph Equivalence which considers additional constraints.

Definition 6 (Restricted DG Equivalence). Let $S$ be a protocol bi-system. Consider the multiset rewrite systems

$$
L=\lceil L(S)\rceil^{D H} \cup I F \cup \mathrm{FRESH}_{S y s} \cup N D^{\prime}
$$

and

$$
R=\lceil R(S)\rceil^{D H} \cup I F \cup \mathrm{FRESH}_{S y s} \cup N D^{\prime} .
$$

We say that $L$ and $R$ are restrictively dependency graph equivalent, written $L(S) \sim_{R D G ; N X} R(S)$, if

- for all dependency graphs dg ensuring restrictions $\mathbf{N X}$ of rules $r \in L \cup R$, the set mirrors $(d g)$ is non-empty and contains dependency graphs for all possible instantiations of new diff-variables.

We call $\sim_{R D G ; N 1, N 2, N 3, N 5^{\prime}, N 5^{\prime \prime}, N 7}$ normal dependency graph equivalence.

As we show below, for protocols that do not multiply exponents and do not introduce products by other means, it is sufficient to check normal dependency graph equivalence. A protocol $P$ is $*$-restricted if, for each of its rules $l-\{a\} \rightarrow r$, (a) $l$ does not contain the function symbols $*,{ }^{\prime},-1$, fst, snd, and dec, and (b) $r$ does not contain the function symbol $*$.

In general, condition (a) prevents protocol rules from pattern matching on reducible function symbols. Condition (b) prevents protocols from directly using multiplication, although repeated exponentiation is still allowed. Note that these restrictions are similar to those of previous work such as $[28,23,12]$ and are not a restriction in practice. Protocols that use multiplication in the group of exponents can usually be specified by using repeated exponentiation. Moreover, protocols that use multiplication in the $\mathrm{DH}$ group, such as MQV [25], cannot be specified anyway, since $*$ denotes multiplication in the group of exponents.

For *-restricted protocols, products that occur in positions that can be extracted by the adversary can always be constructed by the adversary himself from their components. The following lemma shows a generalization of this, namely that the adversary always must construct all input components of a term.

Lemma 1. Let $S$ be a *-restricted protocol, and let $d g \in$ ndgraphs $_{E}(S)$ be a normal dependency graph. If there exists a node with the conclusion $c=K_{e}^{\uparrow}(t)$, then for all $t^{\prime} \in$ inp $(t)$ there are nodes with conclusion $c^{\prime}=K_{e}^{\uparrow}\left(t^{\prime}\right)$ in $d g$.

Proof. By definition, we have that $\operatorname{inp}\left(t^{-1}\right)=i n p(t)$, $\operatorname{inp}\left(\left\langle t_{1}, t_{2}\right\rangle\right)=\operatorname{inp}\left(t_{1}\right) \cup i n p\left(t_{2}\right), \operatorname{inp}\left(t_{1} * t_{2}\right)=\operatorname{inp}\left(t_{1}\right) \cup i n p\left(t_{2}\right)$, and inp $(t)=\{t\}$ otherwise.

- if $t=t^{\prime-1}$, then $c$ is the conclusion of an inverse rule (it cannot be the conclusion of a COERCE rule because of N2), hence there is a premise $\mathrm{K}_{e}^{\uparrow}\left(t^{\prime}\right)$ and by the properties of the dependency graph there must be a matching conclusion $c^{\prime}$.
- if $t=\left\langle t_{1}, t_{2}\right\rangle$, then $c$ is the conclusion of a pairing rule (it cannot be the conclusion of a COERCE rule because of N2), hence there are premises $\mathrm{K}_{e}^{\uparrow}\left(t_{1}\right)$ and $\mathrm{K}_{e}^{\uparrow}\left(t_{2}\right)$, and by the properties of the dependency graph there must be matching conclusions.

- if $t=t_{1} * t_{2}$, then $c$ is the conclusion of a multiplication rule or a COERCE rule. In case of the multiplication rule there are premises $\mathbf{K}_{e}^{\uparrow}\left(t_{1}\right)$ and $\mathbf{K}_{e}^{\uparrow}\left(t_{2}\right)$, and by the properties of the dependency graph there must be matching conclusions. As the protocol is $*$-restricted, in the case of the COERCE rule, $t$ must be a subterm of a term input to the protocol. By the ISEND rule, there must be a corresponding $\mathrm{K}_{e}^{\uparrow}\left(t^{\prime \prime}\right)$ premise such that $t$ is a subterm of $t^{\prime \prime}$. Hence there must be a conclusion $\mathrm{K}_{e}^{\uparrow}(t)$ before. This again could either result from a multiplication rule or a COERCE rule. However, as the protocol is $*$-restricted, this chain needs to eventually end with a multiplication rule, where we can argue as above.

The following lemma shows that mirrors of dependency graphs are actually unique up to equations and instantiations of new diff-variables. Note that in particular there cannot be any differences stemming from diff-terms as in all mirrors they are instantiated in the same way.

Lemma 2. Given a convergent equational theory $E$ and a dependency graph $d g \in$ dgraphs $_{E}(S)$, its mirrors mirrors $(d g)$ are unique up to the equational theory and the instantiation of new diff-variables.

Proof. We show this by induction on the depth of the mirrors.

- In the base case, the dependency graph only consists of a single rule instance without premises. Then, all terms in the conclusion facts can only include functions, constants and new public variables. By the definition of mirrors, all new (non-diff) variables must be instantiated identically, hence the graphs are unique up to the instantiations of new diff-variables.

- Induction step: The root node consists of a rule instance with premises. As we have a correct dependency graph, the premises and conclusions match up to the equational theory (which is convergent, and thus confluent and terminating), and by the induction hypothesis the conclusions are unique up to the equational theory and the instantiations of new diff-variables. Then the terms inside conclusions (if they exist) must be unique up to the equational theory and possible new diff-variables: new (non-diff) variables must be identically instantiated, and (sub)terms from the premises are unique up to the equational theory and the instantiations of new diff-variables.

Now, we show that restricted dependency graph equivalence entails dependency graph equivalence. This is done in two steps: we begin by showing that ignoring dependency graphs violating conditions $\mathbf{N 1}, \mathbf{N 2}, \mathbf{N 3}$ and $\mathbf{N 5}$ ' is sound. In a second step we show that N5" is sound, followed by a proof that the same holds for N7. 


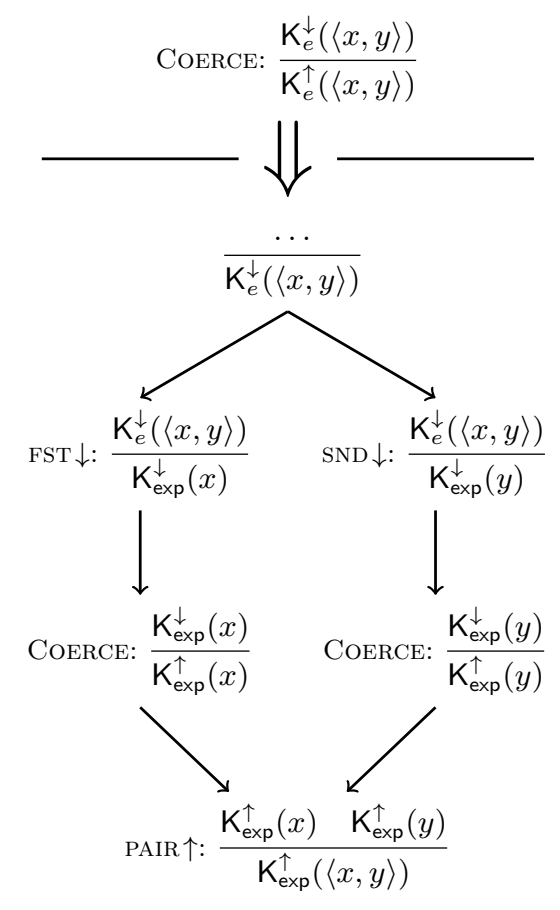

Figure 10: Replacing coerced pairs

$$
\begin{aligned}
& \operatorname{INV} \downarrow: \frac{\mathrm{K}_{e}^{\downarrow}\left(x^{-1}\right)}{\mathrm{K}_{\exp }^{\downarrow}(x)} \\
& \downarrow \\
& \text { CoErce: } \frac{\mathrm{K}_{e}^{\downarrow}\left(x^{-1}\right)}{\mathrm{K}_{e}^{\uparrow}\left(x^{-1}\right)} \Rightarrow \text { CoErcE: } \frac{\mathrm{K}_{\text {exp }}^{\downarrow}(x)}{\mathrm{K}_{\text {exp }}^{\uparrow}(x)} \\
& \begin{array}{c}
\downarrow \\
\operatorname{INV} \uparrow: \frac{\mathrm{K}_{\text {exp }}^{\uparrow}(x)}{\mathrm{K}_{\text {exp }}^{\uparrow}\left(x^{-1}\right)}
\end{array}
\end{aligned}
$$

Figure 11: Replacing coerced inverses

Lemma 3. Let $S$ be a*-restricted protocol bi-system. Then

$$
L(S) \sim_{D G, I F \cup F \mathrm{RESH} \cup N D} R(S)
$$

if and only if

$$
L(S) \sim_{R D G ; N 1, N 2, N 3, N 5^{\prime}} R(S) .
$$

Proof. It is easy to see that $L(S) \sim_{D G, I F \cup \text { Resh } \cup N D} R(S)$ implies $L(S) \sim_{R D G ; N 1, N 2, N 3, N 5^{\prime}} R(S)$ as all restricted normal dependency graphs are dependency graphs.

Assume that $L(S) \sim_{R D G ; N 1, N 2, N 3, N 5^{\prime}} R(S)$. Then

- for all dependency graphs $d g$ ensuring restrictions N1, N2, N3, N5' of rules $r \in L \cup R$ the set of mirrors mirrors $(d g)$ is non-empty and contains dependency graphs for all possible instantiations of new diff-variables.

We must show that the above conditions also hold for nonnormal dependency graphs.

Assume without loss of generality that $d g$ is a non-normal dependency graph of a rule $r \in L$ (the symmetric case is analogous). Then we can transform $d g$ into a normal dependency graph $d g^{\prime}$ by executing the following transformations.

$$
\begin{aligned}
& \operatorname{MULT} \uparrow: \frac{\mathrm{K}_{e_{t}}^{\uparrow}(t) \mathrm{K}_{e_{s}}^{\uparrow}(s)}{\mathrm{K}_{\exp }^{\uparrow}(t * s)} \\
& \mathrm{K}_{e_{1}}^{\uparrow}\left(x_{1}\right) \ldots \mathrm{K}_{e_{n}}^{\uparrow}\left(x_{n}\right) \quad \mathrm{K}_{\exp }^{\uparrow}(t * s) \\
& \operatorname{MULT} \uparrow: \frac{\mathrm{K}_{e_{n+1}}^{\uparrow}\left(x_{n+1}\right) \ldots \mathrm{K}_{e_{l}}^{\uparrow}\left(x_{l}\right)}{\mathrm{K}_{\exp }^{\uparrow}\left(\left(x_{1} * \ldots * x_{n} * t * s\right) *\left(x_{n+1} * \ldots * x_{l}\right)^{-1}\right)} \\
& \downarrow \\
& \mathrm{K}_{e_{1}}^{\uparrow}\left(x_{1}\right) \ldots \mathrm{K}_{e_{n}}^{\uparrow}\left(x_{n}\right) \quad \mathrm{K}_{e_{t}}^{\uparrow}(t) \quad \mathrm{K}_{e_{s}}^{\uparrow}(s) \\
& \operatorname{MULT} \uparrow: \frac{\mathrm{K}_{e_{n+1}}^{\uparrow}\left(x_{n+1}\right) \ldots \mathrm{K}_{e_{l}}^{\uparrow}\left(x_{l}\right)}{\mathrm{K}_{\exp }^{\uparrow}\left(\left(x_{1} * \ldots * x_{n} * t * s\right) *\left(x_{n+1} * \ldots * x_{l}\right)^{-1}\right)}
\end{aligned}
$$

Figure 12: Replacing multiplication instances violating N3
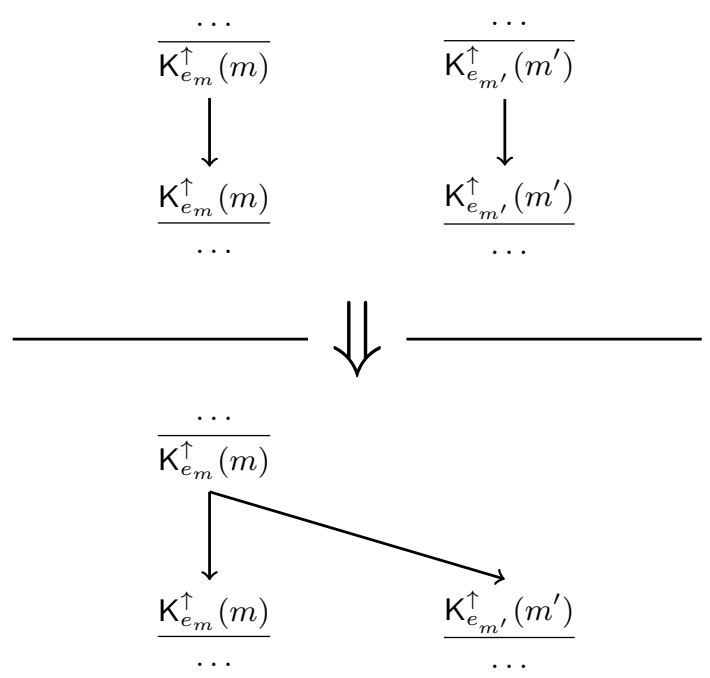

Figure 13: Removing double $\mathbf{K}_{e}^{\uparrow}()$ conclusions

Since $d g$ is non-normal, it must violate (at least) one of the conditions N1-N3 or N5'.

1. If it violates $\mathbf{N} 1$, we replace the rule instances with normalized ones.

2. If there is an instance of COERCE deducing a pair, respectively an inverse (violating N2), we replace this by

(a) instances of the pair deconstruction rules, followed by COERCE instances on the parts, and a pair construction rule (cf. Figure 10), respectively

(b) an instance of the inverse deconstruction rule, followed by a COERCE instance (cf. Figure 11)

If necessary, this must be applied multiple times.

3. If there is an instance of the multiplication rule with a premise fact of the form $\mathrm{K}_{e}^{\uparrow}(t * s)$ (violating N3), this can be replaced by an instance of the multiplication rule where $t$ and $s$ are two distinct premises (cf. Figure 12). By Lemma 1, the necessary premises must exist within $d g$. Again, if necessary, this is applied multiple times. 
4. If there are two distinct conclusions $c$ and $c^{\prime}$ with conclusion facts $\mathrm{K}_{e}^{\uparrow}(m)$ and $\mathrm{K}_{e^{\prime}}^{\uparrow}\left(m^{\prime}\right)$ such that $m={ }_{A C} m^{\prime}$ (violating $\mathbf{N 5}^{\prime}$ ), one of them can be removed, and all edges originating from the removed conclusion can be moved to the remaining conclusion (cf. Figure 13). If necessary, this is applied multiple times.

This gives us a normalized graph $d g^{\prime}$ and, by assumption, we then have that the set of mirrors mirrors $\left(d g^{\prime}\right)$ is non-empty and contains dependency graphs for all possible instantiations of new diff-variables.

Now we need to show that this implies the existence of mirrors for the original graph $d g$. To do this, we will undo the transformations in reverse order and argue why this gives us a correct set of mirrors.

4. We can undo the removal of the second conclusion fact, and also move the edges back. To ensure that the resulting graph is still correct, we however need to check that even in the mirrored graph both conclusion facts still contain the same term (modulo AC).

For both $\mathrm{K}_{e}^{\uparrow}(\cdot)$-facts, consider now their $\mathrm{K}_{e}^{\uparrow}(\cdot)$ deduction subgraph, i.e., the graph originating at their rule instance, and where all branches that are not linked to this via edges that link $\mathrm{K}_{e}^{\uparrow}(\cdot)$-facts, are cut off. Let $d$ denote the depth of the subgraph of $\mathrm{K}_{e}^{\uparrow}(m)$, and $d^{\prime}$ denote the depth of the subgraph of $\mathrm{K}_{e}^{\uparrow}\left(m^{\prime}\right)$. We proceed by induction on $n=\min \left(d, d^{\prime}\right)$.

If $n=0$, then at least one of $\mathrm{K}_{e}^{\uparrow}(m)$ and $\mathrm{K}_{e}^{\uparrow}\left(m^{\prime}\right)$ is a conclusion of the COERCE, PUB, or ONE constructor rules. Note that they cannot be conclusions of the CFresh rule, as this would imply a non-unique fresh value. Therefore we have three cases:

- At least one of them, without loss of generality $\mathbf{K}_{e}^{\uparrow}(m)$, is the conclusion of a COERCE rule: Hence its premise is $\mathbf{K}_{e}^{\downarrow}(m)$, and thus on the original side there is a restricted normal dependency graph with an instance of the equality rule with the premises $\mathrm{K}_{e}^{\downarrow}(m)$ and $\mathrm{K}_{e}^{\uparrow}\left(m^{\prime}\right)$ corresponding to the current dependency graph $d g$. Since we have dependency graph equivalence, this graph also has mirrors on the other side, and this gives us that the instances of $\mathbf{K}_{e}^{\downarrow}(m)$ and $\mathbf{K}_{e}^{\uparrow}\left(m^{\prime}\right)$ contain the same term (modulo AC).

- One of them is a PuB rule: By the previous case, the other one cannot be a conclusion of a COERCE rule. Hence, as all other construction rules cannot result in an equal term, the second fact must also be the conclusion of a PUB rule. Then, by the definition of mirrors, both contain the same new term, and are hence equal.

- One of them is a ONE rule: By the first case, the other one cannot be a conclusion of a COERCE rule. Hence, as all other construction rules cannot result in an equal term, the second fact must also be the conclusion of a ONE rule. Then, by the definition of mirrors, both contain the same term 1 , and are hence equal. Note that there cannot be an instance where one is a PuB rule and one is a OnE rule, as they cannot be equal in $d g$.

If $n>0$ : In this case both facts are conclusions of construction rules with a $\mathrm{K}_{e}^{\uparrow}(\cdot)$ premise. By analyzing all potential rules, we can see that in the original graph $d g$ both facts must be the conclusion of the same rule (otherwise they cannot be equal modulo $\mathrm{AC}$ ), and hence their premises must be equal. Using the induction hypothesis all $\mathrm{K}_{e}^{\uparrow}(\cdot)$ premises are thus equal (modulo $\mathrm{AC}$ ) in the mirrored graph, and hence (by definition of mirrors we have the same rules, and by the previous transformations we have instances of the multiplication rule with the same number of parameters) also the conclusions.

3. If we had to replace instances of the multiplication rule, we can undo the transformation. This gives a correct dependency graph, and it must be in the set of mirrors mirrors $(d g)$ as it has the right structure, rule instances, and variable instantiations.

2. The same argument holds for graphs where we had modify instances of COERCE.

1. We do not need to undo the normalization of terms since we do not care about the instantiations of the rules, as long as they exist.

In a separate lemma, we now prove the second step, the soundness of N5".

Lemma 4. Let $S$ be a *-restricted protocol bi-system. Then we have $L(S) \sim_{R D G ; N 1, N 2, N 3, N 5^{\prime}, N 5^{\prime \prime}} R(S)$ if and only if we have $L(S) \sim_{R D G ; N 1, N 2, N 3, N 5^{\prime}} R(S)$.

Proof. It is easy to see that $L(S) \sim_{R D G ; N 1, N 2, N 3, N 5^{\prime}}$ $R(S)$ implies $L(S) \sim_{R D G ; N 1, N 2, N 3, N 5^{\prime}, N 5^{\prime \prime}} R(S)$ as the second considers fewer graphs due to the additional restriction N5".

Assume that we have $L(S) \sim_{R D G ; N 1, N 2, N 3, N 5^{\prime}, N 5^{\prime \prime}} R(S)$. Then

- for all dependency graphs $d g$ ensuring restrictions N1, N2, N3, N5', N5" of rules $r \in L \cup R$ the set of mirrors mirrors $(d g)$ is non-empty and contains dependency graphs for all possible instantiations of new diff-variables.

We need to show that the above conditions also hold for dependency graphs violating N5".

All graphs violating N5" contain a conclusion $(i, 1)$ with fact $\mathbf{K}_{e}^{\uparrow}(m)$, a conclusion $(j, 1)$ with fact $\mathrm{K}_{e^{\prime}}^{\downarrow}\left(m^{\prime}\right)$ such that $m={ }_{A C} m^{\prime}$ and $i<j$, and an instance $k$ with $(j, 1) \longmapsto$ $(k, 1) \in D$, where $k$ is not an instance of the IEquality rule. Note that $i$ must be either an instance of the COERCE rule, or an instance of a constructor. This is easy to see by analyzing the message deduction rules.

Assume without loss of generality that $d g$ is a dependency graph of a rule $r \in L$ (the symmetric case is analogous) violating N5". We proceed by induction on the number of rule instances inside $d g$ violating N5" (we call the rule instance $j$ with conclusion $\mathrm{K}_{e^{\prime}}^{\downarrow}\left(m^{\prime}\right)$ the instance "violating" N5").

In the base case, we have no rule instance $j$ violating N5", and we are trivially done.

In the induction step, we choose the rule instance $j$ violating N5" such that $j$ is maximal among all rule instances violating N5". By assumption, there is a $k$ such that $(j, 1) \longmapsto$ $(k, 1) \in D$ and $k$ is not an instance of the IEquality. Moreover, by N5', $k$ cannot be an instance of the COERCE rule. Thus it must be an instance of a deconstruction or exponentiation rule. 
As explained above, the corresponding rule instance $i$ must either be an instance of the COERCE rule, or an instance of a constructor.

1. If $i$ is an instance of the COERCE rule, then there must be a conclusion $(h, 1)$ with fact $\mathrm{K}_{e^{\prime \prime}}^{\downarrow}\left(m^{\prime \prime}\right)$ and $m^{\prime}={ }_{A C}$ $m^{\prime \prime}$. Hence we can replace the edge $(j, 1) \longmapsto(k, 1)$ by an edge $(h, 1) \longmapsto(k, 1)$. The resulting dependency graph $d g^{\prime}$ is still correct and ensures N5". Thus, by $L(S) \sim_{R D G ; N 1, N 2, N 3, N 5^{\prime}, N 5^{\prime \prime}} R(S)$ we have all necessary mirrors. On these mirrors we can undo the transformation by resetting the edge to its original origin. This yields a correct dependency graph, as a graph where we replace $j$ with an instance of the IEquality rule comparing $\mathrm{K}_{e^{\prime}}^{\downarrow}\left(m^{\prime}\right)$ (conclusion $(i, 1)$ ) and $\mathrm{K}_{e}^{\uparrow}(m)$ (conclusion $(j, 1)$ ) also has its mirrors and Lemma 2 holds. Note that this argument is correct even if $k$ is the root of $d g$.

2. If $i$ is an instance of a constructor rule, we distinguish two cases:

(a) In the first case, $k$ is the instance of a destructor rule. Then $k$ 's conclusion is identical to one of $i$ 's premises. This means that there must be an $i^{\prime}$ with a $\mathrm{K}_{e}^{\uparrow}()$ conclusion for each premise, and notably for the one that contains the same term as $k$ 's conclusion.

As we chose $j$ maximal, all rule instances $l$ depending on $k$ 's conclusion (if they exist) must be instances of the IEquality rule, otherwise they would also contradict N5" and $l>k$. By N5' $\mathrm{K}_{e}^{\uparrow}()$ conclusions are unique, hence all instances $l$ must compare $k$ 's conclusion to $i^{\prime}$ 's conclusion.

Now consider the dependency graph $d g^{\prime}$ obtained by removing $k$ and the instances $l$ (if they exist), and adding an instance of the IEquality rule comparing conclusion $(j, 1)$ to $(i, 1)$. Then $j$ no longer contradicts N5", and by the induction hypothesis we have all necessary mirrors.

In all of these mirrors we can undo the transformation by adding a suitable instance of the destructor also used in $k$, and, if necessary, IEquality rule instances to mirror $l$. The destructor $k$ can be applied as its premise is equal to the result of the matching constructor $i^{\prime}$. Moreover, since $\mathrm{K}_{e}^{\uparrow}()$ conclusions are unique, other $\mathbf{K}_{e}^{\uparrow}()$ premises of the destructor (e.g., the key in case of a decryption) can only originate in the premises of rule $i$, and thus must also match in the mirrors. Finally, the equalities also hold because they compare the conclusion of the destructor to the premise of the constructor. Hence the resulting dependency graphs are correct.

(b) In the second case, $k$ is the instance of an exponentiation rule. Then all rule instances $l$ with $(k, 1) \longmapsto(l, 1)$ must be instances of the IEquality or COERCE rule, as we cannot have a second exponentiation rule and no other destructors match. If there are instances of the IEquality rule, we can argue as in the first case.

If there is an instance of the COERCE rule, its conclusion can also be directly derived from the preceding $\mathbf{K}_{e}^{\uparrow}()$ conclusions. Firstly, for any instance of the exponentiation rule, the terms in the exponent are a subset of the input components of

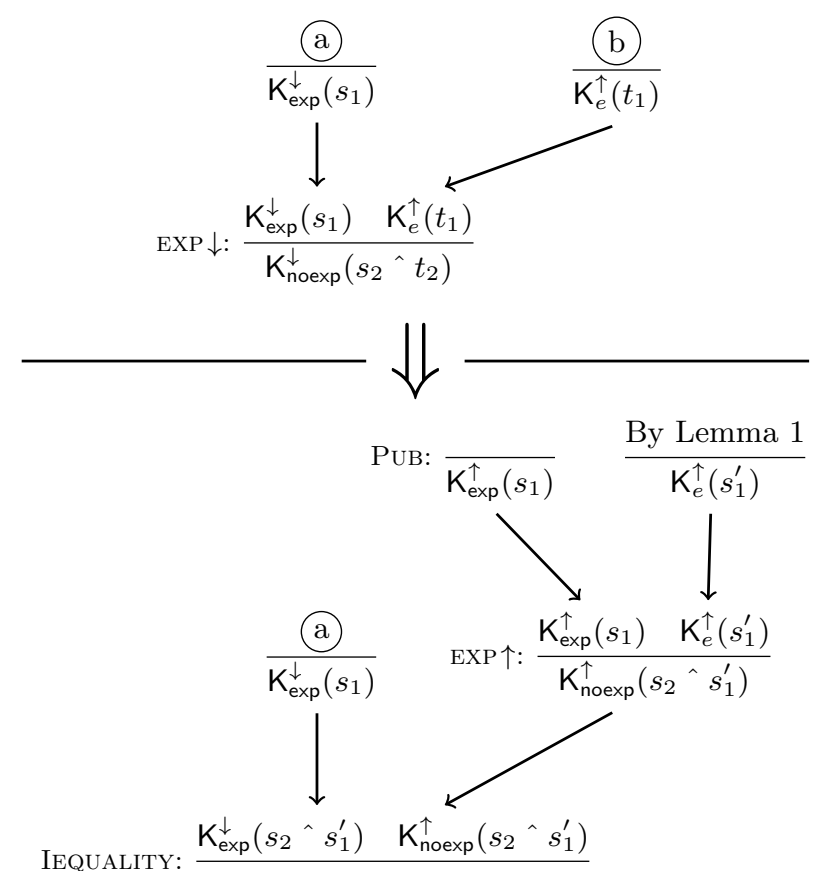

Figure 14: Proof of Lemma 5, case (a)

the exponent of the first $K_{e}^{\downarrow}()$-premise and the input components of the second $K_{e}^{\uparrow}()$-premise. Secondly, the $\mathrm{K}_{e}^{\downarrow}()$ premise results from a constructor, thus there are $\mathrm{K}_{e}^{\uparrow}()$-conclusions for the base and by Lemma 1 for all input components of the exponent. Also, by Lemma 1 there are $\mathrm{K}_{e}^{\uparrow}()$-conclusions for all input components of the exponentiation rule's $\mathrm{K}_{e}^{\uparrow}()$ premise. Thus, the result of the COERCE rule instance can also be deducted from previous $\mathrm{K}_{e}^{\uparrow}()$ facts using an instance of a multiplication rule and an instance of the exponentiation constructor.

The accordingly modified graph now has one instance less violating N5", and by the induction hypothesis we have the necessary mirrors. In all mirrors we can undo the transformation using Lemma 2 and the same arguments as above.

Now we prove the soundness of N7.

Lemma 5. Let $S$ be $a *$-restricted protocol bi-system. Then we have $L(S) \sim_{R D G ; N 1, N 2, N 3, N 5^{\prime}, N 5^{\prime \prime}, N 7} R(S)$ if and only

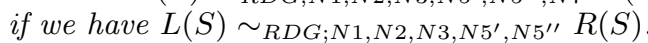

Proof. It is easy to see that $L(S) \sim_{R D G ; N 1, N 2, N 3, N 5^{\prime}, N 5^{\prime \prime}}$

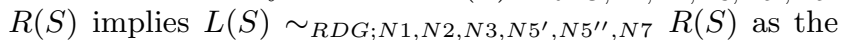
second considers fewer graphs due to the additional restriction N7.

Assume that we have $L(S) \sim_{R D G ; N 1, N 2, N 3, N 5^{\prime}, N 5^{\prime \prime}, N 7} R(S)$. Then

- for all dependency graphs $d g$ ensuring restrictions N1, N2, N3, N5', N7 of rules $r \in L \cup R$ the set of mirrors mirrors $(d g)$ is non-empty and contains dependency graphs for all possible instantiations of new diff-variables. 

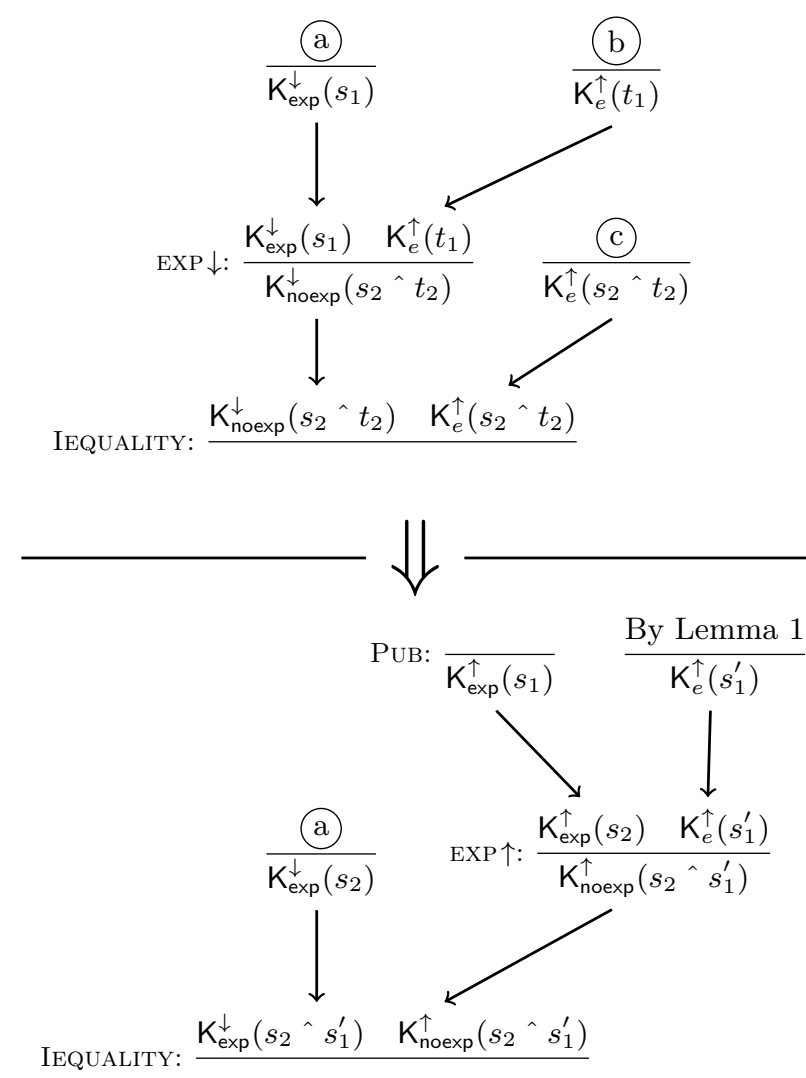

Figure 15: Proof of Lemma 5, case (b)

We need to show that the above conditions also hold for dependency graphs violating N7.

First note that for all nodes violating N7, i.e.,

$$
\mathrm{K}_{\exp }^{\downarrow}\left(s_{1}\right), \mathrm{K}_{e}^{\uparrow}\left(t_{1}\right)-[\} \rightarrow \mathrm{K}_{\text {noexp }}^{\downarrow}\left(s_{2} \wedge t_{2}\right)
$$

such that $s_{2}$ is of sort $p u b, i n p\left(t_{2}\right) \subseteq i n p\left(t_{1}\right)$ we have the following properties:

- they are instances of the exponentiation deconstruction rule,

- $s_{1}=s_{2}^{s_{1}^{\prime}}$, and

- $\operatorname{inp}\left(s_{1}^{\prime}\right) \subseteq \operatorname{inp}\left(t_{1}\right)$.

This is easy to see by analyzing all message deduction rules. It means, in particular, that the intruder can deduce the fact $\mathrm{K}_{\exp }^{\uparrow}\left(s_{1}\right)$ as $s_{2}$ is of sort $p u b$, and that the intruder knows all input components of $t_{1}$ by Lemma 1 .

Assume without loss of generality that $d g$ is a dependency graph of a rule $r \in L$ (the symmetric case is analogous) violating N7. We proceed by induction on the number of rule instances inside $d g$ violating $\mathbf{N 7}$. In the base case, we distinguish three sub-cases.

1. There is only one rule instance violating $\mathbf{N 7}$, namely in the root of $d g$ :

Consider now a graph where we replace the root node with an instance of the equality rule. To obtain a correct dependency graph, the graph (a) above $\mathrm{K}_{\exp }^{\downarrow}\left(s_{1}\right)$ remains unchanged, the rest of the graph needs to be extended with an instance of the exponentiation rule with
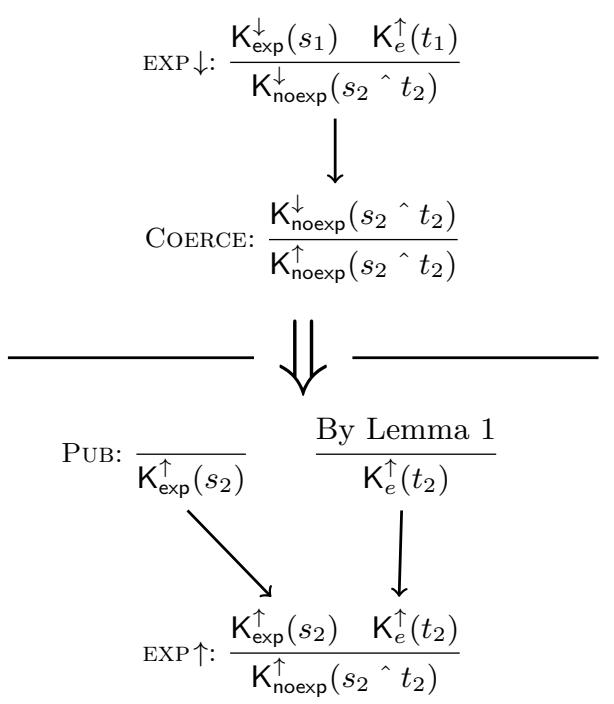

Figure 16: Proof of Lemma 5, case (c)

the premises $\mathrm{K}_{\exp }^{\uparrow}\left(s_{2}\right)$ and $\mathrm{K}_{e}^{\downarrow}\left(s_{1}^{\prime}\right)$ to match the equality, as illustrated by Figure 14. As $\mathrm{K}_{\exp }^{\uparrow}\left(s_{2}\right)$ can be deduced from the public constructor rule and by Lemma 1 , such a graph exists. Moreover, it is restrictively normal, and thus by $L(S) \sim_{R D G ; N 1, N 2, N 3, N 5^{\prime}, N 7} R(S)$ we have all necessary mirrors. Finally, on all graphs in the set of mirrors we can undo the transformation. Note that the input components of $t_{1}$ might not all exist in the new graph. However, we can take the subgraph b that was used to deduce them in $d g$ and take its mirror for the same instantiation of new diff-variables. By Lemma 2 this graph must be identical (up to the equational theory) to the previous graph on the common subparts, hence we can combine both. This must yield a correct dependency graph, and thus we obtain the set of mirrors mirrors $(d g)$.

2. There is only one rule instance violating N7, namely just above the root of $d g$, which is an instance of the equality rule:

Consider now a graph where we essentially do the same transformation as above: we remove the exponentiation deconstruction rule and add an equality rule comparing the $\mathrm{K}_{\exp }^{\downarrow}\left(s_{1}\right)$ to its deduction from the input terms of $t_{1}$, as shown in Figure 15. The resulting dependency graph is restrictively normal, and thus by $L(S) \sim_{R D G ; N 1, N 2, N 3, N 5^{\prime}, N 7} R(S)$ we have all necessary mirrors. Finally, on all graphs in the set of mirrors we can undo the transformation. Again, the input components of $t_{1}$ might not all exist in the new graph. However, we can take the subgraph that was used to deduce them in $d g$ and take its mirror for the same instantiation of new diff-variables. By Lemma 2, this graph must be identical (up to the equational theory) to the previous graph on the common subparts, hence we can combine both. Similarly, for the second premise $\mathrm{K}_{\text {noexp }}^{\uparrow}\left(s_{2}{ }^{\wedge} t_{2}\right)$ of the original equality rule instance, we can use the same argument to show that the graph (C) deducing it has the necessary mirrors, which are compatible with the other graphs. To see that the resulting 
fact ensures the required equality, we consider the two cases: either $\mathrm{K}_{\text {noexp }}^{\uparrow}\left(s_{2}{ }^{\wedge} t_{2}\right)$ is the conclusion of an exponentiation construction rule, or it is the conclusion of a COERCE rule.

(a) In the first case, its premises are (1) the public value $s_{2}$, which must be identically instantiated by the construction of the mirrors, and (2) $t_{2}$, i.e., a subset of the input components of $t_{1}$. By N5' and Lemma 1, this fact must be derived from the same fact as the $\mathrm{K}_{e}^{\uparrow}\left(t_{1}\right)$ premise fact of the exponentiation rule.

(b) In the second case, it is the conclusion of a COERCE rule. In this case we can construct another dependency graph from the graph for $\mathrm{K}_{\text {noexp }}^{\uparrow}\left(s_{2}{ }^{\wedge} t_{2}\right)$, namely a graph where we compare $\mathrm{K}_{\text {noexp }}^{\downarrow}\left(s_{2}{ }^{\wedge} t_{2}\right)$ to its deduction from the public value $s_{2}$ and the input components $t_{2}$ of $t_{1}$. By $L(S) \sim_{R D G ; N 1, N 2, N 3, N 5^{\prime}, N 7}$ $R(S)$ we have all necessary mirrors, which again gives us the equality for all required graphs.

Thus, we are sure in both cases that the equality holds, hence combining all graphs yields a correct dependency graph, and we obtain the set of mirrors mirrors $(d g)$.

3 . In all other cases we have one instance of the exponentiation deconstruction rules violating $\mathbf{N 7}$, where the resulting $\mathrm{K}_{\text {noexp }}^{\downarrow}\left(s_{2}{ }^{\wedge} t_{2}\right)$ fact is immediately transformed into a $\mathrm{K}_{\text {noexp }}^{\uparrow}\left(s_{2} t_{2}\right)$ fact using an instance of the COERCE rule (see Figure 16). Then we can replace both rule instances (exponentiation and COERCE) by an instance of the exponentiation construction rule, where $s_{2}$ and $t_{2}$ are derived from the intruder's knowledge facts (possible by Lemma 1).

The resulting graph ensures N7, and hence we have the necessary mirrors. We can undo the transformation and obtain mirrors for the original graph $d g$. To see that we still have a correct dependency graph, we can use the same argument as above: we build a dependency graph with an IEQUALITY rule as the root and the $\mathrm{K}_{\text {noexp }}^{\downarrow}\left(s_{2} \wedge t_{2}\right)$ from the exponentiation deconstruction rule and the $\mathrm{K}_{\text {noexp }}^{\uparrow}\left(s_{2} \wedge t_{2}\right)$ fact from the exponentiation construction rule. By the previous case, we have the necessary mirrors for this case, which gives us the equality of the terms by Lemma 2 .

The induction step is analogous to the last case of the base case: we replace one instance where the result of the exponentiation deconstruction rule is immediately coerced with an instance of the exponentiation construction rule. Then we can apply the induction hypothesis, and conclude as above.

Taking both lemmas together, we obtain the desired result.

THEOREM 3. Let $S$ be a*-restricted protocol bi-system. Then we have $L(S) \sim_{D G, I F \cup F \mathrm{resh} \cup N D} R(S)$ if and only if we have $L(S) \sim_{R D G ; N 1, N 2, N 3, N 5^{\prime}, N 5^{\prime \prime}, N 7} R(S)$.

Proof. By Lemmas 3, 4 and 5.

\section{A.3 Constraint Solving}

Building on Theorem 3, we can implement the algorithm in Figure 4 in TAMARIN by using the existing constraint solving (see the extended version of [28] for details) to compute all (restricted) normal dependency graphs of a rule. For this, we add a unique action to each rule, and search for all cases where this action occurs.

Note that we had to modify the constraint solving to reflect the new restricted normal form conditions (cf. Figure 17). We removed the rule enforcing condition N6, and weakened the rule enforcing N5 to only ensure N5' and N5". Moreover we modified rules $\mathrm{DG} 2_{2, \uparrow e}$ and $\mathrm{DG} 2_{2, \uparrow i}$ to also solve trivial $\mathrm{K}_{e}^{\uparrow}(m)$ premises. For us, it is important whether even trivial premises were deduced from a protocol output or not. Moreover, solving all premises allows us to directly extract dependency graphs from the solved constraints without having to instantiate the open ones.

Similarly to [28], we say that $(d g, \theta)$ is a $P$-model of $\Gamma$, if $d g$ is a restricted normal dependency graph for $P$ and $(d g, \theta) \vDash$ $\Gamma$. A $P$-solution of $\Gamma$ is a restricted normal dependency graph $d g$ for $P$ such that there is a valuation $\theta$ with $(d g, \theta) \vDash$ $\Gamma$.

LEMMA 6. The modified constraint solving is sound and complete, i.e., for each constraint reduction step the set of $P$-solutions is unchanged.

Proof. The proof is essentially the same as the proof in the extended version of [28], except we do not have the case for N6 any more and need adapt the cases N5', N5 ", DG2 $2, \uparrow e$ and DG2 $2, \uparrow i$. Considering completeness, we have the following new or different cases (all other cases are as in [28]):

N5' From the rule's side-condition, we have $i: r i \in \Gamma$, and $i^{\prime}: r i^{\prime} \in \Gamma$ such that $\mathrm{K}_{e}^{\uparrow}(t)={ }_{A C} \operatorname{concs}(r i)_{1}$, and $\mathrm{K}_{e^{\prime}}^{\uparrow}(t)={ }_{A C} \operatorname{concs}\left(r i^{\prime}\right)_{1}$. Hence, $\theta(i), \theta\left(i^{\prime}\right) \in\{1, \ldots,|I|\}$, ri $\theta={ }_{A C} I_{\theta(i)}, r i^{\prime} \theta={ }_{A C} I_{\theta\left(i^{\prime}\right)}, \operatorname{concs}\left(I_{\theta(i)}\right)_{1}={ }_{A C} \mathrm{~K}_{e}^{\uparrow}(t \theta)$, and $\operatorname{concs}\left(I_{\theta\left(i^{\prime}\right)}\right)_{1}={ }_{A C} \mathrm{~K}_{e^{\prime}}^{\uparrow}(t \theta)$. Moreover, we have $(\theta(i), 1)=\left(\theta\left(i^{\prime}\right), 1\right)$ from $\mathbf{N 5}$ ', which concludes this case.

N5" From the rule's side-condition, we have $i:\left(l-\left[\rightarrow \rightarrow \mathrm{K}_{e}^{\uparrow}(t)\right)\right.$, $j:\left(l^{\prime}-\left[\rightarrow \mathrm{K}_{e}^{\downarrow}\left(t^{\prime}\right)\right), k: r i,(j, 1) \rightarrow(k, 1), i \lessdot j \in \Gamma\right.$ such that $r i \notin$ ginsts(IEQUALITY) and $t={ }_{A C} t^{\prime}$. Hence, $\theta(i), \theta(j), \theta(k) \in\{1, \ldots,|I|\}$, moreover $\theta(i)<\theta(j)$, $r i \theta \in \operatorname{ginsts}$ (COERCE) and $t \theta={ }_{A C} t^{\prime}$, so by the definition of $-\rightarrow$ in any solution there exists a rule instance with index $k^{\prime}$ and $(\theta(j), 1) \longmapsto\left(k^{\prime}, 1\right)$ such that $k^{\prime}$ is either an instance of a destructor rule or an instance of the COERCE rule, which contradicts property N5" and thus concludes this case.

DG2 $2, \uparrow e$ From the rule's side-condition, we have $j: r i^{\prime} \in \Gamma, v \in$ $\left\{1, \ldots,\left|\operatorname{prems}\left(r i^{\prime}\right)\right|\right\}$ such that $p=(j, v), \operatorname{prems}\left(r i^{\prime}\right)_{v}=$ $\mathrm{K}_{e}^{\uparrow}(m)$, and $\{m\}=\operatorname{inp}(m)$. Hence, $\theta(j) \in\{1, \ldots,|I|\}$, $r i^{\prime} \theta={ }_{A C} I_{\theta(j)}$, and $\operatorname{prems}\left(I_{\theta(j)}\right)_{v}={ }_{A C} \mathbf{K}_{e}^{\uparrow}(m) \theta$. From DG1-2, we obtain $k \in\{1, \ldots,|I|\}$ and $u \in\left\{1, \ldots,\left|\operatorname{concs}\left(I_{k}\right)\right|\right\}$ such that $(k, u) \longmapsto(\theta(j), v) \in D$ and $\operatorname{concs}\left(I_{k}\right)_{u}={ }_{A C}$ $\mathrm{K}_{e}^{\uparrow}(m) \theta$. As $\{m\}=i n p(m), m$ is provided by a construction rule in $N D^{c-e x p l}$, thus there is $r u \in N D^{c-e x p l}$ and a grounding substitution $\sigma$ such that $I_{k}=r u \sigma$.

We construct a model of the constraint system

$$
\Gamma^{\prime}=\{i: r u \rho,(i, u) \longmapsto p\} \cup \Gamma
$$

where $\rho$ is a fresh renaming of $r u$ with respect to $\Gamma$. The constraint system $\Gamma^{\prime}$ occurs in the right-hand-side of the constraint reduction rule. The structure

$$
\left(d g, \theta[i \mapsto k][\rho(x) \mapsto \sigma(x)]_{x \in \operatorname{dom}(\sigma)}\right)
$$




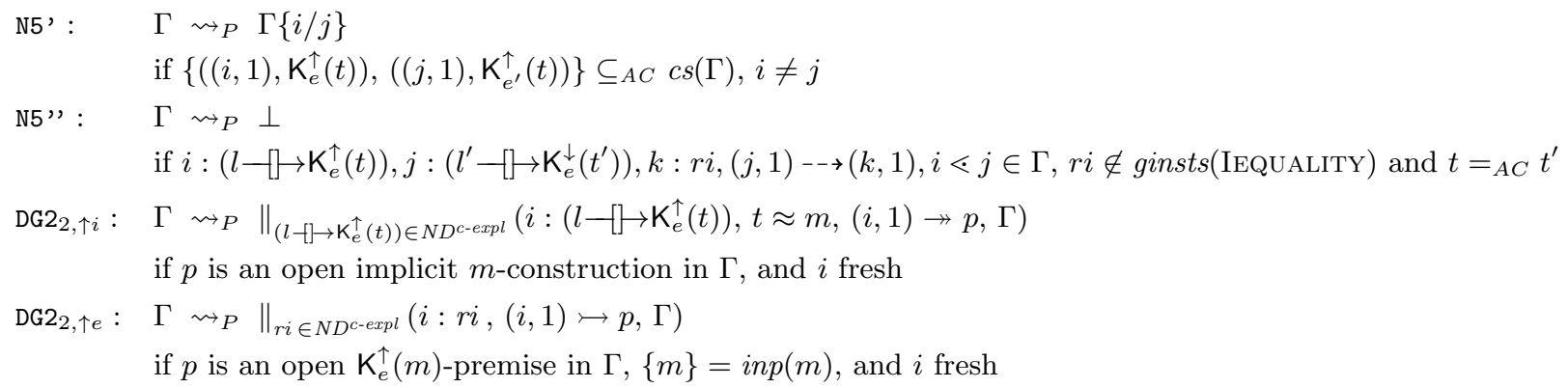

Figure 17: Modified constraint-reduction rules.

is a $P$-model of $\Gamma$ as only the valuation of fresh variables is changed. It is also a model of $i: r u \rho$ and $(i, u) \longmapsto p$. Thus, it is a model of $\Gamma^{\prime}$.

DG2 $2, \uparrow i$ From the rule's side-condition, we have $j: r i \in \Gamma$, $v \in\{1, \ldots,|\operatorname{prems}(r i)|\}, s \in \mathcal{T}$, and $m \in \operatorname{inp}(s) \backslash$ $(\{s\})$ such that $p=(j, v)$ and prems $(r i)_{v}=\mathrm{K}_{e}^{\uparrow}(s)$. Hence, $\theta(j) \in\{1, \ldots,|I|\}$ and $r i \theta={ }_{A C} I_{\theta(j)}$. Moreover, prems $\left(I_{\theta}(j)\right)_{v}={ }_{A C} \mathrm{~K}_{e}^{\uparrow}(s \theta)$ and $m \theta \in_{A C}$ inp $(s \theta) \backslash$ $\{s \theta\}$. From [28, Extended version, Lemma 3], we obtain $k \in\{1, \ldots,|I|\}, r u \in N D^{c-e x p l}$, a substitution $\sigma$ grounding for $r u, e^{\prime}$, and $m \in \mathcal{M}$ such that $I_{k}=r u \sigma$, $\operatorname{concs}(r u \sigma)_{1}=\mathrm{K}_{e^{\prime}}^{\uparrow}\left(m^{\prime}\right), m \theta={ }_{A C} m^{\prime}$, and $(k, 1) \rightarrow_{d g}$ $(\theta(j), v)$. We construct a $P$-model for the constraint system

$$
\Gamma^{\prime}=\left\{i: l \rho-\{\} \rightarrow \mathrm{K}_{e^{\prime}}^{\uparrow}(t \rho), t \rho \approx m,(i, 1) \rightarrow p\right\} \cup \Gamma
$$

where $r u=l-[\} \rightarrow \mathrm{K}_{e^{\prime}}^{\uparrow}(t)$ and $\rho$ is a fresh renaming for $r u$ with respect to $\Gamma$. The constraint system $\Gamma^{\prime}$ occurs in the right-hand-side of the constraint reduction rule. The structure

$$
\left(d g, \theta[i \mapsto k][\rho(x) \mapsto \sigma(x)]_{x \in \operatorname{dom}(\sigma)}\right)
$$

is a model of $i: l \rho-[\} \rightarrow \mathrm{K}_{e^{\prime}}^{\uparrow}(t \rho), t \rho \approx m,(i, 1) \rightarrow d g$ $p$, and $\Gamma$, as only fresh variables are renamed. This concludes this case.

Considering soundness, no changes to the proof are necessary.

Using Lemma 6, we can now show that the implemented algorithm is sound.

THEOREM 4. The algorithm in Figure 4 is sound, i.e., when it returns "verification successful", we have $(L(S) \cup$ $\left.\operatorname{FRESH}_{S y s}\right) \approx_{\mathrm{FRESH}_{E n v} \cup N D}\left(R(S) \cup \mathrm{FRESH}_{S y s}\right)$.

Proof. We have $L(S) \sim_{R D G ; N 1, N 2, N 3, N 5^{\prime}, N 7} R(S)$ if the algorithm returns "verification successful" by the soundness and completeness of the constraint solving (Lemma 6). By Theorem 3 this implies $L(S) \sim_{D G, I F \cup \text { RRESH } \cup N D} R(S)$, and therefore by Theorem $2\left(L(S) \cup \mathrm{FRESH}_{S y s}\right) \approx_{\mathrm{FRESH}_{E n v} \cup N D}$ $\left(R(S) \cup \mathrm{FRESH}_{\text {Sys }}\right)$.

\section{A.4 Optimizations}

We call a dependency graph unfinished when it contains premises that do not have incoming edges (called open premises, violating DG2), but ensures DG1, DG3, and DG4.

To improve the performance of our algorithm, we implemented the following optimization allowing us to ignore $\mathrm{in}$ dependent open premises during the constraint solving. We say that an open premise fact of an unfinished dependency graph is independent if both

- all its terms are different variables and

- all these variables neither occur in other branches of the graph nor in other facts of the same rule.

Intuitively, if a premise is independent, it can be generated on the left-hand side system (LHS) if and only if it can be generated on the right-hand side system (RHS), as all terms are arbitrary and dependency graph equivalence ensures that we can derive an instance of a rule (an thus its conclusion facts) on the LHS if and only if it can be derived on the RHS. This allows us to abort constraint solving if all open premises are independent and the current (unfinished) dependency graph has the required mirrors.

We call an unfinished dependency graph trivial if all its open premises are trivial. Note that the definition of mirrors can be directly applied to unfinished trivial dependency graphs. A completion $d g^{\prime}$ of an unfinished dependency graph $d g$ is a complete dependency graph $d g^{\prime}$ that contains $d g$.

The following lemma shows the soundness of our optimization.

Lemma 7. Let $S$ be a protocol bi-system. Consider the multiset rewrite systems $L=[L(S)]^{D H} \cup I F \cup \mathrm{FRESH}_{\text {Sys }} \cup N D^{\prime}$ and $R=[R(S)\rceil^{D H} \cup I F \cup \mathrm{FRESH}_{\text {Sys }} \cup N D^{\prime}$. For each rule $r \in L \cup R$, let $D G_{r}$ be a set of (unfinished trivial) restricted normal dependency graphs such that their completions cover all dependency graphs of the rules $r$. If for all $r$ and all $d g \in$ $D G_{r}$ the set mirrors $(d g)$ is non-empty and contains dependency graphs for all possible instantiations of the trivial open premises and for all possible instantiations of the new diffvariables, then we have $L(S) \sim_{R D G ; N 1, N 2, N 3, N 5^{\prime}, N 7} R(S)$.

Proof. We need to show that for all completions of all unfinished trivial dependency graphs $d g \in D G_{r}$ the set mirrors $(d g)$ is non-empty and contains dependency graphs for all possible instantiations of new diff-variables. Let $d g$ be such an unfinished trivial dependency graph and $d g_{r}^{\prime}$ be one of its completions. We must show that mirrors $\left(d g^{\prime}\right)$ contains instances for possible instantiations of new diff-variables. Let $P$ denote all open premises in $d g$. For all $p \in P$ let $d g_{p}^{\prime}$ denote the sub-dependency graph of $d g^{\prime}$ that roots at $p$. For all $d g_{p}^{\prime}$ the set of mirrors $\left(d g_{p}^{\prime}\right)$ is non-empty and contains dependency graphs for all possible instantiations of the new diff-variables. For each possible instantiations of the new diff-variables, the union of all these dependency graphs together with a dependency graph $m d g \in \operatorname{mirrors}(d g)$ whose instantiations of the trivial open premises matches the instances of the graphs $d g_{P}^{\prime}$ gives a correct dependency graph by Lemma 2, which concludes the proof. 
Finally the optimization enables us to ignore certain rules when checking dependency graph equivalence. The construction, COERCE, communication and exponentiation rules in $N D$, the FRESH rules, and all constructors generated from the equational theory only contain independent facts as premises, hence we need not check them. We only need to check the protocol rules, and the destructors from $N D$ and from the equational theory. In practice, a protocol's initialization rules also often only have trivial premises, but this must be checked for each protocol. Our implementation automatically recognizes the situation where it can abort for protocol rules, whereas the construction, COERCE, communication and exponentiation rules in $N D$, the FRESH rules, and all constructors generated from the equational theory are not even analyzed as they always fall in this category. 